Check for updates

Cite this: Mater. Adv., 2020, 1,3122

Received 22nd August 2020, Accepted 29th October 2020

DOI: 10.1039/d0ma00632g

rsc.li/materials-advances

\title{
Solid-state fluorescent carbon dots: quenching resistance strategies, high quantum efficiency control, multicolor tuning, and applications
}

\author{
Junli Wang, ${ }^{a}$ Yongzhen Yang (D) *a and Xuguang Liu (D) ${ }^{b}$
}

\begin{abstract}
As a new member of the carbon family, carbon dots (CDs) not only inherit the advantages of good biocompatibility and abundant raw material sources from traditional carbon materials, but also exhibit outstanding luminescence characteristics, such as tunable emissive wavelength and light bleaching resistance. Thus, they show great application prospects in many fields. However, CDs may suffer from serious aggregation-induced fluorescence quenching and lack of high quantum efficiency and tunable full color solid-state fluorescence, which hinder their large-scale application. In this review, the recent advances in the solid-state fluorescence properties of CDs are summarized, with special emphasis on the strategies for quenching resistance, methods for high quantum efficiency, and methods for multicolor tuning. The strategies for obtaining quenching-resistant CDs include the dispersion of CDs in situ and post processing, and the preparation of CDs with uniform structure. Solid-state fluorescent CDs with high quantum efficiency can be obtained by controlling the matrix type and CD concentration, doping heteroatoms, and adding salt crystals in situ and reducing the carbonization degree. Solid-state multi-color CDs are realized using three methods, including the effective dispersion of multicolor CDs, gradient concentration dispersion of concentration-induced multicolor emissive CDs and fluorescence origin (i.e., core size, surface states and molecular states) tuning of self-quenching-resistant CDs. The applications of solid-state fluorescent CDs in fingerprint identification, photoelectric device and visible light communication are also outlined. Finally, their challenges and prospects in the future are presented.
\end{abstract}

\section{Introduction}

Despite the earlier discovery of fluorescence from onion-like fullerenes by another group, in 2006, Sun et al. ${ }^{1}$ discovered one class of novel carbon materials with fluorescence characteristics and quantum size, and named them carbon dots (CDs). Since then, the research field of CDs has thrived. As emerging fluorescent nanomaterials, CDs not only inherit the advantages of traditional carbon materials, such as wide range of raw materials and good biocompatibility, but also have excellent optical properties, including adjustable luminescence wavelength and good optical stability, showing promising potential in the fields of photoelectric devices, biomedicine and environmental monitoring. Therefore, they have attracted extensive attention and in-depth study from researchers. CDs are a general term for graphene quantum dots, carbon nanodots

\footnotetext{
${ }^{a}$ Key Laboratory of Interface Science and Engineering in Advanced Materials, (Taiyuan University of Technology), Ministry of Education, Taiyuan 030024, China. E-mail: yyztyut@126.com

${ }^{b}$ Institute for New Carbon Materials, Taiyuan University of Technology, Taiyuan 030024, China
}

and polymer dots (PDs). ${ }^{2}$ Generally, CDs are regarded as zerodimensional spherical particles less than $10 \mathrm{~nm}$, composed of a carbon core and surface functional groups. ${ }^{3,4}$ The specific structure of CDs is determined by reaction precursors and parameters. Specifically, the carbon core of CDs may be graphite-like or amorphous, with various surface functional groups, such as $-\mathrm{OH},-\mathrm{COOH}, \mathrm{C}=\mathrm{O}, \mathrm{C}=\mathrm{N}, \mathrm{NH}_{2}$ and $\mathrm{C}-\mathrm{O}-\mathrm{C} .^{5}$

The methods for the preparation of CDs can be roughly classified into "top-down" and "bottom-up". For the "topdown" methods, CDs are formed by stripping nanoparticles from carbon skeletons, including arc discharge, ${ }^{6}$ laser etching, ${ }^{7,8}$ and electrochemical oxidation. ${ }^{9,10}$ In the case of the "bottom-up" methods, CDs are prepared via the dehydration and carbonization of precursor molecules, which mainly include combustion/pyrolysis, ${ }^{11}$ microwave method, ${ }^{12}$ and hydro-/solvothermal method. ${ }^{13,14}$ Among them, the hydro-/ solvothermal and microwave methods have become the conventional methods to synthesize CDs because of their simple and environmentally friendly synthetic process and the high fluorescence intensity of the resulting CDs. ${ }^{15}$ In the early stage of the study of CDs, using the above synthetic methods, the luminescence of CDs was mostly concentrated in the 
short-wavelength regions. However, in recent years, the emission of CDs has been gradually extended to the orange, red, and even near-infrared region and multicolor adjustable luminescence of CDs has been realized by regulating the reaction conditions. In the past few years, the photoluminescence (PL) mechanisms of CDs have been explored and summarized as the following four: carbon core state influenced the quantum size effect or conjugated $\pi$-domain, surface state determined by the carbon core and connected functional groups, molecule state, and crosslinking enhancement effect. ${ }^{2}$

Most reports about the luminescence characteristics and mechanism of CDs focus on CD solutions and there are a few researches on the fluorescence property of CDs in the solid state. In fact, the fluorescence intensity of most CDs greatly or even completely decreases in solid-state powder or film because of excessive fluorescence resonance energy transfer (FRET) or direct $\pi-\pi$ interactions. $^{16,17}$ The solid-state fluorescence quenching of CDs seriously hinders their large-scale application in the fields of photoelectric devices, ${ }^{18}$ fingerprint identification, fluorescent ink, security labels, and other solid-state luminescence fields. Therefore, it is very important to explore quenching resistance strategies for CDs. Furthermore, high efficiency and multi-color solid-state luminescent CDs exhibit broad prospects in the fields of display and lighting, ${ }^{19}$ but the realization of highly emissive solid-state full color CDs still faces great challenges. At present, the solid-state quantum efficiency of CDs is still low. In addition, the emission peaks of solid-state luminescent CDs are mainly concentrated in the blue, green, and yellow regions, although there are some reports on orange- and red-emissive solid-state CDs. There is lack of reports on full-color adjustable solid-state fluorescent CDs with high quantum efficiency. Therefore, it is of great significance to realize highly emissive solid-state fluorescence CDs and their multicolor regulation.

Obtaining multi-color adjustable solid-state luminescent CDs with quantum efficiency and exploring their application in the solid-state luminescent field will give theoretical and practical support for realizing solid-state luminescence in CDs. In this review, a comprehensive overview about solid-state fluorescent CDs is given. Firstly, the quenching resistance strategies of solid-state fluorescent CDs are discussed. Secondly, the methods for high quantum efficiency of solid-state fluorescent CDs are reviewed. Thirdly, the multicolor regulation methods of solid-state fluorescent CDs are described. Then, the application fields of solid-state fluorescent CDs are summarized. Finally, the development prospect of solid-state fluorescent CDs is presented.

\section{The strategies for quenching- resistant CDs}

Induced by $\pi-\pi$ stacking or excessive nonradiative energy transfer, CDs always suffer from serious self-quenching in the solid state. Therefore, in order to realize solid-state fluorescence in CDs, their self-quenching must be effectively inhibited. The strategies for quenching resistance include avoiding direct contact between CD nanoparticles by using a physical method to uniformly disperse the CDs in some matrix or using a chemical method to form a large steric structure between the CDs (seen as Sections 2.1-2.3), and synthesizing CDs with uniform structure (seen as Section 2.4), both of which can inhibit nonradiative energy transfer.

The steric hindrance between CDs can be formed by a "twostep method" or "one-step method". The "two-step method" means that CDs are first prepared and then physically embedded in a matrix, such as polymer, salt crystal, and starch, to realize solid-state PL (seen as Section 2.1). The "one-step method" means the solid-state PL of CDs can be directly realized without post-processing, where it is necessary to regulate the carbon core and surface functional groups of CDs by controlling precursors and their carbonization degree. For example, using chemicals with a long-chain structure or salt crystal as processors to synthesize CDs (seen as Section 2.2), or forming a crosslinked structure in CDs (seen as Section 2.3), thus spatial distance is in situ formed in CDs, which is beneficial to inhibit the formation of strong $\pi-\pi$ interaction and further realize solid-state PL. The detailed descriptions are given as follows.

\subsection{Dispersing CDs in a polymer or solid matrix by the two- step method}

As one of the common matrices, polymers have dual functions. They can act as a dispersant to increase the distance between CD nanoparticles and further inhibit the solid-state quenching of CDs. They can also work as a film-forming agent to endow CDs with film-forming characteristics, which are beneficial in many applications. Common polymer matrices include epoxy resin, polyvinyl alcohol (PVA), polymethyl methacrylate (PMMA), polyvinylpyrrolidone (PVP), ${ }^{20}$ polyethylene glycol (PEG), polystyrene (PS), and polyethyleneimine (PEI). Long et al. $^{21}$ synthesized CDs from glucose in HF solution, which exhibited bright yellow fluorescence in solution but no fluorescence after drying on paper. When PEG was applied to the paper, the CDs showed yellow fluorescence with a quantum yield (QY) of $10.4 \%$ (Fig. 1a). This is because the PEG chains wrap around the surface of the CDs, increasing the distance between the CD nanoparticles, which inhibits FRET and significantly enhances the emission of the CDs.

Besides polymer matrices, some solid substances can also act as a dispersion matrix. Sun et al. ${ }^{22}$ developed a novel solid dispersion matrix, starch, and further investigated the effect of starch content on the solid-state luminescence property of CDs. This method provides a novel way to research the solid-state luminescence of CDs (Fig. 1b). They found that starch can adsorb CDs due to their effective dispersion on the surface of starch, and consequently, FRET and aggregation-induced PL quenching can be suppressed. To improve the UV and thermal stability of CD-based composite phosphors, a salt crystal was developed as a matrix. Kim et al. ${ }^{23}$ embedded CDs in $\mathrm{NaCl}, \mathrm{KCl}$, and $\mathrm{KBr}$ crystals to realize solid-state PL in CDs. They found that the $\mathrm{NaCl}$ matrix provides $\mathrm{CDs}$ with good protection against UV irradiation, and under $250 \mathrm{~h}$ of UV exposure, $70 \%$ of the 
a
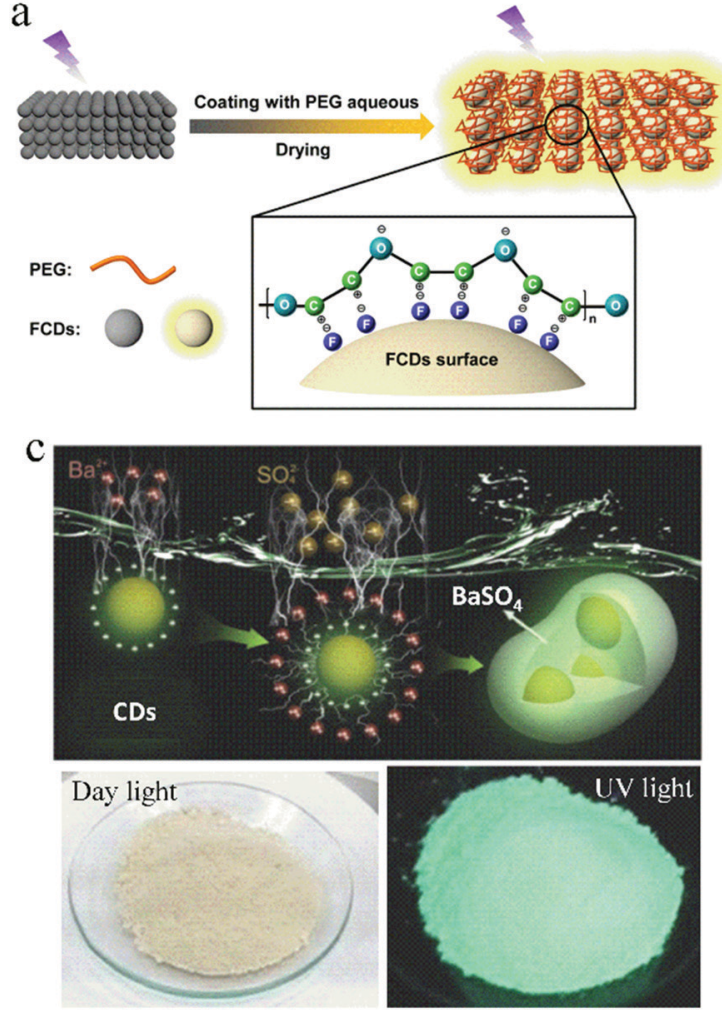

b
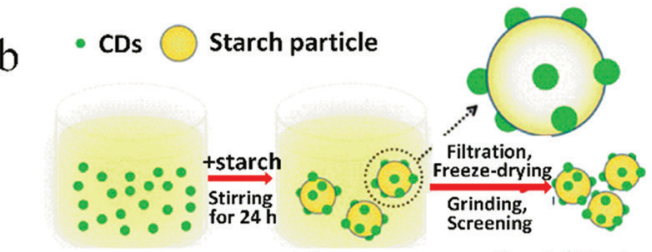

$\mathrm{CD}$ aqueous solution Starch/CD composites Starch/CD phosphors
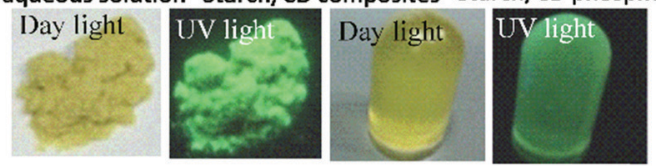

d
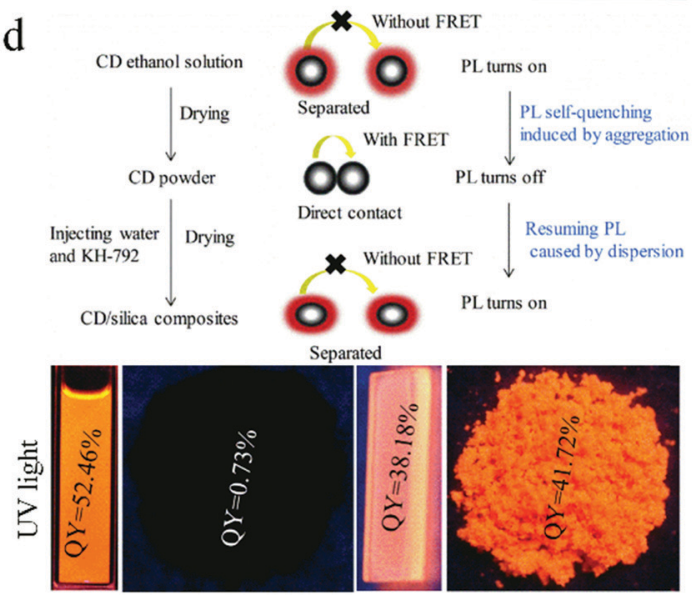

Fig. 1 (a) Proposed mechanism of PEG-switched fluorescence emission of solid-state CDs. Reproduced with permission from ref. 21. Copyright 2017, the American Chemical Society. (b) Schematic illustration of the formation mechanism of starch/CDs and their photographs. Reproduced with permission from ref. 22. Copyright 2014, The Royal Society of Chemistry. (c) Schematic of the fabrication of $\mathrm{CDs}^{\circ} \mathrm{aBaSO} \mathrm{B}_{4}$ and its photographs. Reproduced with permission from ref. 24. Copyright 2016, John Wiley and Sons. (d) (top) Schematic of the self-quenching in solid-state CDs and the resistance against solid-state self-quenching of $\mathrm{CDs}$ by embedding into $\mathrm{SiO}_{2}$ matrix. (bottom) Photographs of $\mathrm{CD}$ solution, $\mathrm{CD}$ powder, $\mathrm{CD} / \mathrm{SiO} 2$ film, and $\mathrm{CD} / \mathrm{SiO}_{2}$ powder under UV light. Reproduced with permission from ref. 25. Copyright 2017 Elsevier.

initial fluorescence intensity of the $\mathrm{CD} / \mathrm{NaCl}$ composites was retained. The $\mathrm{KBr}$ crystal gave $\mathrm{CDs}$ with excellent thermal stability and about $80 \%$ PL retention after being heated at $80{ }^{\circ} \mathrm{C}$ for $250 \mathrm{~h}$. Zhou et $a .^{24}$ reported the electrostatic assembly-guided synthesis of $\mathrm{CDs} / \mathrm{BaSO}_{4}$ hybrid phosphors with strong luminescence (Fig. 1c). $\mathrm{BaSO}_{4}$ is a type of environmentally friendly and low-cost material. When it attached on the surface of $\mathrm{CDs}$ to form $\mathrm{CD} / \mathrm{BaSO}_{4}$ hybrid phosphors, the aggregation of CDs could be effectively avoided. The $\mathrm{CDs} / \mathrm{BaSO}_{4}$ hybrid phosphors exhibited strong solid-state luminescence and high stability, and remarkable resistance to strong acid/ alkali and common organic solvents. The fluorescence intensity of $\mathrm{CDs} / \mathrm{BaSO}_{4}$ was maintained at $92 \%$ after UV irradiation at $300{ }^{\circ} \mathrm{C}$ for $30 \mathrm{~min}$.

Wang et $a l^{25}$ reported a method of physically embedding CDs into an $\mathrm{SiO}_{2}$ matrix to endow CDs with solid-state orange emission and film-forming characteristics. $\mathrm{N}$-(3-(Trimethoxysilyl)propyl)ethylenediamine (KH-792) was injected into the $\mathrm{CD}$ solution to form a $\mathrm{CD} / \mathrm{SiO}_{2}$ film and powder. The QY of the $\mathrm{CD} /$ $\mathrm{SiO}_{2}$ film and powder was $38.2 \%$ and $41.7 \%$, respectively, which is considerably higher than that of pure solid-state CDs, i.e., $0.73 \%$. A mechanism for the PL quenching in the solid-state CDs and PL realized in the $\mathrm{CD} / \mathrm{SiO}_{2}$ composites was proposed. Specifically, in CD powder, nanoparticles directly contact with each other to induce excessive FRET and further PL quenching. While in the $\mathrm{CD} / \mathrm{SiO}_{2}$ composites, the distance between the $\mathrm{CDs}$ is increased because of the uniform dispersion of $\mathrm{CDs}_{\text {in }} \mathrm{SiO}_{2}$, and consequently, FRET is avoided, PL quenching is inhibited, and the solid-state PL of CDs is realized (Fig. 1d). Similarly, Kipnusu et al. ${ }^{26}$ fabricated CDs via the one-step femtosecond pulsed laser irradiation of a single precursor (2-aminopyrimidine5-boronic acid) in solution and realized high solid-state PL with a QY of $46 \%$ by dispersing the CDs into nanoporous $\mathrm{SiO}_{2}$.

The above methods to prepare solid-state luminescent CDs belong to the "two-step method", where CDs are synthesized and then dispersed into various matrices by physical methods and the matrices act as the medium to dilute CDs, without changing the structure of the CDs. Once the proportion of CDs in the matrix is high, PL quenching still occurs, and thus the solid-state quenching of CDs is not eliminated in by the "two-step method". ${ }^{27}$ However, in practical application, highperformance photoelectric devices require CDs with strong fluorescence output, which must be met by a high concentration loading of CDs. Therefore, it is urgent to develop "onestep methods" to synthesize solid-state fluorescent CDs. At present, the main "one-step methods" to realize solid-state PL in CDs are using polymer, long-chain alkyl materials, coupling agents, and salts as raw materials, or forming large 
electrostatic repulsive forces, hydrogen bonds, and crossinglinking structures in CDs, to realize the in situ dispersion of CDs.

\subsection{In situ dispersing CDs using of polymers, long chain alkyl materials, coupling agents and salts}

A common strategy to directly realize solid-state luminescence in CDs is to use polymers or materials with long chain structures as raw materials to in situ synthesize CDs with steric hindrance. There have been several reports on the use of polymers as precursors to obtain solid-state luminescent CDs. ${ }^{28}$ As a precursor, polymers can avoid fluorescence quenching of CDs from the following two aspects, decreasing the conjugated region of CDs to avoid strong $\pi-\pi$ interaction and forming a crosslinked polymer network structure to make CDs selfdisperse, which weakens the strong intermolecular interaction. PVA, PVP, PS, and PEI are widely used as precursors.

$\mathrm{Xu}$ et $a .^{29}$ prepared CDs with bright blue emission both in solution and powder form using CA and PVP as raw materials via a one-step hydrothermal method. The self-quenching resistant properties of CDs were due to the steric hindrance of PVP on the CD surface, which avoids the direct contact between the CD nanoparticles. Liu et al. ${ }^{30}$ used CA and PEI with a branched chain structure as the precursors to obtain CDs, which exhibited solid-state green emission with a QY of $26.0 \%$. In this case, the solid-state quenching of CDs was inhibited by the PEI branch chain on the CD surface. Chen et al. ${ }^{31}$ fabricated CDs with solid-state green emission via the one-pot hydrothermal treatment of PVA and ethanediamine. As shown in Fig. 2a, the effective steric hindrance of PVA keeps the CD nanoparticles at appropriate intervals, which may be the most important factor for their solid-state luminescence. A further study on the fluorescence of the $\mathrm{CD}$ solution showed that the well-dispersed $\mathrm{CD}$ solution exhibited blue emission, and as the concentration of the CD solution increased from 0.3 to $30 \mathrm{mg} \mathrm{mL}^{-1}$ until it became powder, the fluorescence peak of CDs gradually shifted from 414 to $525 \mathrm{~nm}$. The yellow emission of the CDs was caused by their aggregation because the shorter distance between the CD nanoparticles than $R_{0}$, combined with the spectral overlap of absorption and emission spectra, induced FRET or re-absorption and red-shift of the emission. This study provides a new theoretical foundation for the preparation of solid-state luminescent CDs. In addition, they realized the preparation of multicolor solid-state luminescent CDs with PVA as the carbon source and changing the dopant (Fig. 2b). In detail, tetraethylenepentamine and diethylenetriamine were used as nitrogen dopants to prepare d-CD and t-CD, respectively, with yellow and orange fluorescence in the solid state.

Similar with polymers, long-chain alkanes also contribute to the formation of a highly cross-linked polymer network structure in CDs, which can reduce the formation of cyclic molecules and conjugated systems and decrease the interaction of CDs. Furthermore, the long-chain groups of the alkanes can segregate CDs to realize solid-state luminescence. Jiang et al. ${ }^{32}$ synthesized white-emitting CDs via the pyrolysis of polyethylene glycol sorbitol monolaurate with long-chain alkyl groups and applied them to unlabeled latent fingerprint imaging. The results showed that the presence of long-chain alkyl groups on the surface of the CDs effectively inhibited the solid-state quenching and gave bright white luminescence under UV irradiation in both solution and powder. The QY of the CD powder was $2.0 \%$, which is comparable to that $(2.1 \%)$ in solution.

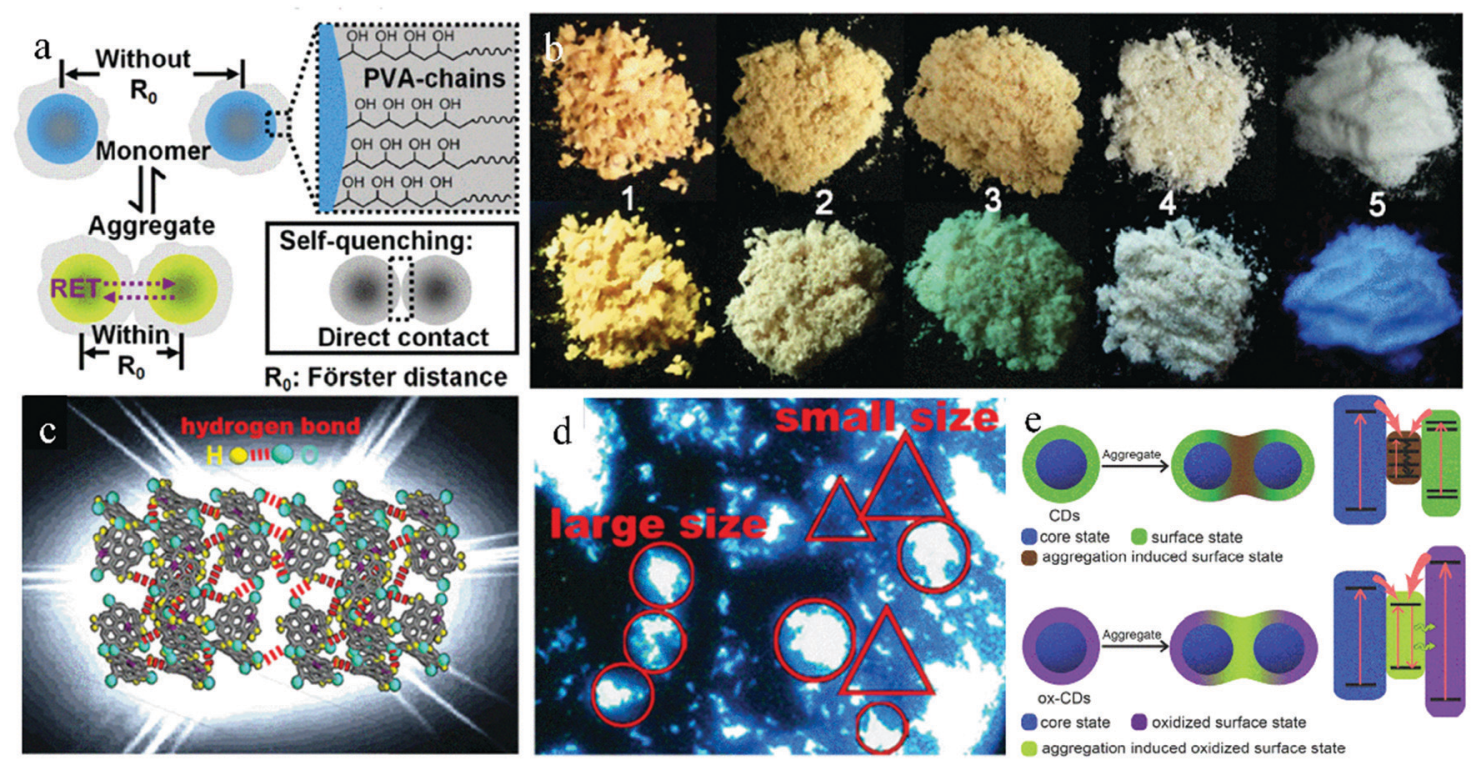

Fig. 2 (a) Schematic of self-quenching-resistance of CD powder. Inset: Schematic of self-quenching of many other luminescent species. (b) Photographs of d-CD (No. 1), t-CD (No. 2), CD (No. 3), PVA (No. 4), and CD/starch composite (No. 5) powder. Reproduced with permission from ref. 31. Copyright 2015, John Wiley and Sons. (c) Structural diagrams of BWCD powder. (d) Optical microscopy image of solid BWCDs in alcohol suspension. Reproduced with permission from ref. 40. Copyright 2019, The Royal Society of Chemistry. (e) Schematic of CDs and ox-CDs in the dispersed and aggregated states; frames on the right show possible band-energy structures and quenching processes of CDs in the aggregated state and recombination processes of ox-CDs in the aggregated state. Reproduced with permission from ref. 46. Copyright 2018, John Wiley and Sons 
Using a silane coupling agent as the precursor also realized the in situ dispersion of CDs and inhibited their solid-state self-quenching. During the synthetic process, the amino group in the silane coupling agent reacts with organic acids to form CDs through dehydration, polymerization, and carbonization. Importantly, the alkoxy group in the silane coupling agent can be hydrolyzed or alcoholized to form a large $\mathrm{SiO}_{2}$ network structure on the surface of the CDs, which can weaken the strong supramolecular interaction between the CD nanoparticles, avoid strong $\pi-\pi$ interaction, and realize solid-state luminescence in CDs. Wang et al. $^{33}$ synthesized silanefunctionalized CDs for the first time using CA and $\mathrm{N}$-(amino-ethyl)amino propylene-trimethoxysilane (AEAPMS) as raw materials. The CDs showed bright blue emission in solution and film. Zheng et al. ${ }^{34}$ prepared blue light-emitting CDs using CA and KH-792 as raw materials via a one-step microwave-assisted hydrothermal method. The solid-state QY of the CDs was up to $65.8 \%$, which was 2.5 -fold that $(25.6 \%)$ of their solution. He et al..$^{35}$ also used CA and AEAPMS as raw materials to obtain red fluorescent CDs with a QY of $9.6 \%$ in the solid state via a solvothermal reaction in acetone. Further, $\mathrm{CD} /$ starch composite phosphors with double peak emission of blue light and orange light were constructed by dispersing CDs in starch.

By using small salt molecules as raw materials and in situ embedding CDs in salt crystals, some researchers synthesized solid-state luminescent CDs. Shen et al. ${ }^{36}$ prepared CDs with solid-state green fluorescence and QY of $21.6 \%$ via a one-step synthetic method using urea and sodium citrate as precursors and $N, N$-dimethyl formamide (DMF) as the solvent. During the synthesis, CDs are in situ embedded in sodium citrate crystals with steric hindrance, which can effectively restrain the luminescence quenching of the CDs. Similarly, Wang et al. ${ }^{37}$ used glutathione and sodium citrate to obtain CDs via a one-step hydrothermal method, which exhibited solid-state blue-green emission with a maximum at $500 \mathrm{~nm}$ and QY of $34.1 \%$. Using calcium citrate and urea as raw materials, Xu et al. ${ }^{38}$ synthesized CDs with solid-state green emission centered at $520 \mathrm{~nm}$ and QY of $40 \%$ via a one-step microwave method. Zheng et al. ${ }^{39}$ synthesized CDs with phthalic acid and formamide as raw materials and glycerol as the solvent. During the reaction, the CDs were in situ embedded into the crystal of phthalimide to realize yellow solid-state fluorescence emission with a QY of $20.3 \%$.

\subsection{Synthesizing CDs with strong electrostatic repulsion, hydrogen bonding or low conjugation}

Forming hydrogen bonds or electrostatic interactions on the surface of CDs is another strategy in the category of "one-step method" to realize solid-state luminescent CDs. Meng et al. ${ }^{40}$ developed a strategy of hydrogen bonding to realize ultrabroadband white-emissive CDs (BWCDs) with a QY of $10 \%$ in the solid state. BWCDs were prepared via a hydrothermal method at $170{ }^{\circ} \mathrm{C}$ with guanidine carbonate and potassium dihydrogen phosphate as raw materials. There were abundant $\mathrm{C}=\mathrm{O}$ and $-\mathrm{NH}_{2}$ functional groups on the surface of BWCDs. Compared with the wavenumber of $\mathrm{C}=\mathrm{O}$ and $\mathrm{NH}_{2}$ in $\mathrm{CD}$ solution, that in powder shifted to a lower frequency. Based on these results, they proposed the hydrogen bonding between $\mathrm{C}=\mathrm{O}$ and $-\mathrm{NH}_{2}$ in the solid-state BWCDs (Fig. 2c). Furthermore, the BWCD dispersion showed blue emission, which gradually broadened and redshifted with an increase in concentration, indicating that the white-light emission of solid-state BWCDs was caused by aggregation. As revealed by the fluorescence microscopy image (Fig. 2d), the CDs with a small size exhibited good dispersion and blue emission, while the aggregates with a large size exhibited white light emission. Therefore, at high concentration, aggregates were formed and induced solid-state white-light emission.

Hydrogen bond and electrostatic interaction can also synergistically realize solid-state fluorescence in CDs. Shen et al. ${ }^{41}$ developed an N/B doping strategy to realize the solid-state green fluorescence of CDs with a QY of $18 \%$ using boric acid and ethylenediamine as raw materials via a one-step hydrothermal method. They proposed that the introduction of $\mathrm{B}$ atoms may contribute to the solid-state luminescence of the CDs from following aspects. On the one hand, electrondeficient $\mathrm{B}$ atoms can inhibit the charge transfer of CDs. On the other hand, the $\mathrm{B}-\mathrm{OH}$ functional groups on the surface of CDs can form hydrogen bonds, which can keep the CD powder in a dispersed state, similar to that in solution. In addition, the existence of a large number of negatively charged functional groups on the surface of the CDs result in particles with strong electrostatic repulsion, which inhibits the excessive aggregation of the CDs. The above factors resulted in the CDs maintaining the dispersion state in powder and realizing solid-state fluorescence. Using CA as the carbon source, and ethylenediamine and boric acid as dopants, Choi et al. ${ }^{42}$ synthesized blue emission CDs with a QY of $80.8 \%$ in solution and up to $67.6 \%$ in powder via a one-step microwave method. For comparison, CA was used as the carbon source and ethylenediamine as the nitrogen dopant without boric acid to prepare CDs, which also had blue emission with a QY of $40.2 \%$ in solution, but only $1 \%$ in powder. These results prove that the introduction of B is beneficial for the solid-state luminescence of CDs.

The low conjugation structure of CDs also inhibits their solid-state quenching. Different from most CDs with good crystallization, PDs are composed of a cross-linked matrix and fluorophores, without an obvious lattice structure. Thus, the synthesis of PDs with a low conjugation degree is an effective method to suppress the solid-state quenching of CDs. Under neutral and acidic conditions, using CA and $p$-amino salicylic acid as the raw materials and the hydrothermal method, Feng et $a l^{43}$ synthesized yellow- and orange-emissive PDs with a solid-state QY of $1.04 \%$ and $0.42 \%$, respectively.

In all the above methods, the CDs realized solid-state PL by effective separation due to steric hindrance. From the level of "spatial distance of donor-acceptor in the energy transfer process", the non-radiative energy transfer between different surface states or energy levels of CDs is suppressed, and then the fluorescence quenching of CDs is inhibited. In fact, if CDs possess uniform surface states or particle sizes, and thus there is no donor-acceptor to transfer energy, the occurrence of non-radiative energy transfer between $\mathrm{CD}$ nanoparticles can 
be suppressed from the level of "whether or not donor-acceptor energy transfer exists", and the fluorescence quenching of CDs can also be inhibited.

\subsection{Synthesizing CDs with a uniform structure}

CDs are generally considered to be composed of a carbon core and surface functional groups. When CDs aggregate, the interaction between their different surface states can promote the occurrence of solid-state quenching effect. Therefore, pure surface states in CDs are beneficial to greatly reduce the donoracceptor interaction and achieve solid-state luminescence in CDs. Similarly, a uniform particle size in CDs will lead to uniform energy levels and avoid the non-radiative energy transfer of the CDs, and thus inhibit fluorescence quenching, which also is conducive to the realization of solid-state luminescence. Li et al $^{44}$ reported that the pure surface state of CDs is one of key factors to realize their solid-state fluorescence. With PVA as a single reaction raw material and $\mathrm{NaOH}$ for accelerating carbonization and polymerization, CDs were prepared with only $-\mathrm{OH}$ on their surface via the hydrothermal method at $250{ }^{\circ} \mathrm{C}$ for $6 \mathrm{~h}$. The $\mathrm{CDs}$ exhibited green-yellow fluorescence emission in both the solution and solid states. Li et $a{ }^{45}$ found that the inhomogeneous surface charge distribution of CDs drives their self-assembly in the aggregated state, resulting in the fluorescence quenching of CD powder. Thus, based on the above research, Zhou et al. ${ }^{46}$ developed a type of surface treatment to realize solid luminescence in CDs. The original CDs were prepared from CA and ammonia water via a microwave method, which underwent fluorescence quenching in the solid state. After oxidation treatment with $\mathrm{H}_{2} \mathrm{O}_{2}$, oxidized CDs (ox-CDs) were obtained with strong solidstate green emission and QY of 25\%. Further analysis showed that ox-CDs possessed a more uniform surface charge distribution than the original CDs. The surface coupling state of the aggregated original CDs opened the non-radiation channel, and thus the aggregation induced luminescence quenching in the original CDs. However, that of aggregated ox-CDs induced the radiation channel, which led to solid-state green fluorescence emission (Fig. 2e). Therefore, synthesizing CDs with a uniform surface state is an effective method to realize solidstate luminescence in CDs.

Zhang et $a l .{ }^{47}$ proposed that uniform particle size is also a key factor to realize solid-state fluorescence in CDs. In the presence of $\mathrm{KCl}$, CDs were prepared with $\mathrm{CA}$ and L-cysteine as raw materials via a one-step microwave method. The unique foamed structure of the CDs effectively inhibited their excessive growth, giving them uniform size and enabling them to emit bright solid-state yellow luminescence with a QY of up to $65 \%$.

\section{The methods for high quantum efficiency of solid-state fluorescent CDs}

The high quantum efficiency of solid-state fluorescent CDs is a prerequisite for outstanding brightness and luminous efficiency in CD-based devices and clear fingerprint latent images, which is very important for practical application. One way to achieve this goal is to give solid-state luminescence characteristics to CDs that have high fluorescence in solution using the above-mentioned anti-quenching strategy (dispersing CDs into polymer or solid-state matrix). Consequently, the high QY of CDs can be retained in the solid state under the protection of the dispersion matrix, and further high efficiency solid-state fluorescent CDs can be achieved. The realization methods for highly efficient fluorescent CDs in the solution state have been described in many reviews, ${ }^{48,49}$ and thus will not be repeated here. Instead, the factors influencing the solid-state luminescence of CDs in the process of dispersing CDs into a matrix are summarized, that is, the matrix type and CD concentration (as shown in Section 3.1). Another way is to regulate the structure of CDs, and thus CDs can directly realize highly efficient solidstate fluorescence without an additional dispersion process. The main methods can be summarized as follows: doping heteroatoms (as shown in Section 3.2), and adding salt crystals in situ and reducing the carbonization degree (as shown in Section 3.3).

\subsection{Controlling the matrix type and CD concentration}

As mentioned above, the solid-state fluorescence of CDs can be achieved by dispersing them in a matrix. In fact, the type of matrix will affect the solid-state fluorescence of CDs through their interaction, and even the same CDs embedded in a different matrix will present different emissions. Therefore, a proper dispersion matrix is very important for high efficiency solid-state fluorescence in CDs. In addition, a too low concentration loading of CDs cannot output a high intensity fluorescence emission because of the small amount of fluorescent molecules, while an excessive concentration loading of CDs may induce their fluorescence quenching because of their poor dispersion. Therefore, controlling the concentration of CDs in the matrix is also meaningful for high solid-state fluorescence in CDs.

The effect of matrix on the solid-state fluorescence of CDs has been investigated in some studies. Deng et al. ${ }^{50}$ systematically studied the solid-state fluorescence of the same blue emissive CDs dropped on different substrates. As shown in Fig. 3a, the CDs show bright blue emission when dropped on PVA, polyacrylamide (PAM), PEG, cotton, and cellulose paper, while no fluorescence emission was observed on tetrafluoroethylene, ITO, and Si substrates. This is because the blue emission of the solid-state CDs depends on the polar groups of substrates, that is, the blue light emission of the solid-state CDs is caused by the interaction between the $\mathrm{C}=\mathrm{O}$ on the $\mathrm{CD}$ surface and the polar groups in the substrates. Jin et al. ${ }^{51}$ used a charged environment-friendly cellulose derivative to separate CD nanoparticles by electrostatic interaction, effectively inhibiting the fluorescence quenching of the CDs. The negatively charged cellulose was used to combine with positively charged blue emissive CDs to form the blue emissive CDs@cellulose derivative powder with a high solid-state QY of $71.5 \%$. In contrast, the positively charged cellulose was selected to combine with negatively charged green and red emissive CDs to 

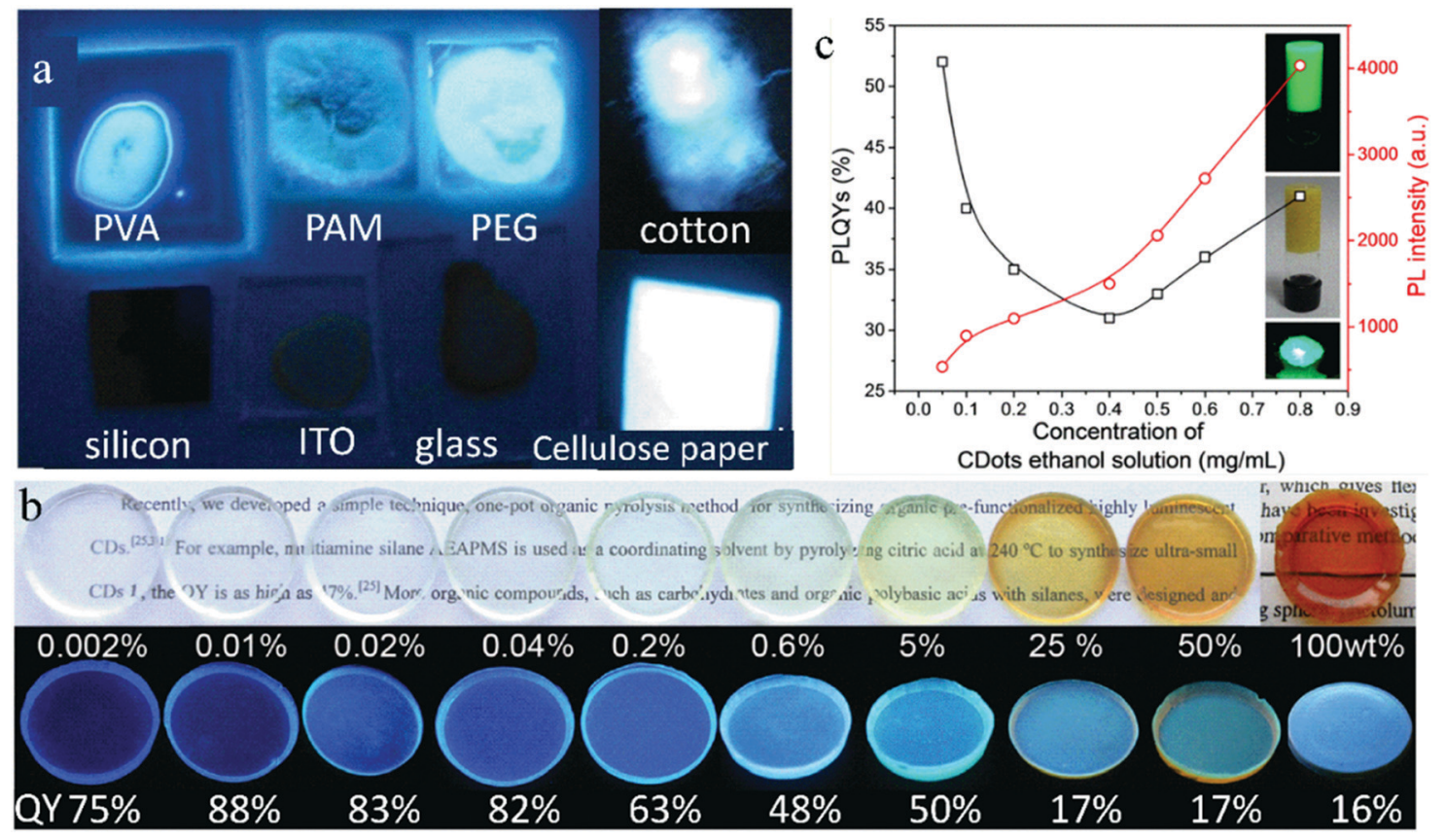

Fig. 3 (a) Photographs of CDs on various substrates of PVA, PAM, PEG20000, cotton, silicon, ITO, glass, and cellulose paper under UV light. Reproduced with permission from ref. 50. Copyright 2014, The Royal Society of Chemistry. (b) Optical photographs of CD-gel glass monoliths doped with various ratios of CDs upon visible light (top) and $365 \mathrm{~nm}$ UV illumination (bottom). Reproduced with permission from ref. 52. Copyright 2012, John Wiley and Sons. (c) Variation in QY (open black squares) and PL intensity (open red circles) of the CD@silica composites versus the concentration of CD ethanol solution. The insets are photographs of the CD@silica gel under UV light and sunlight, and CD asilica xerogel under UV light. Reproduced with permission from ref. 53. Copyright 2017, the American Chemical Society.

form green and red emissive CDs@cellulose derivative powders with a solid-state QY of $11.3 \%$ and $30.4 \%$, respectively.

Meanwhile, the effect of CD concentration in the dispersion matrix on their solid-state fluorescence was also studied by some researchers. Xie et $a .^{52}$ synthesized CDs with filmforming ability using organosilane as the raw material through a one-step pyrolysis. Then, the CDs were dispersed in silane to form various $\mathrm{CDs@SiO}{ }_{2}$ gel glass. The mass ratio of CDs in the gel glass was adjusted from 0 to $100 \mathrm{wt} \%$ The solid-state QY of the pure CDs was only $16 \%$. However, their solid-state QY increased by loading them in the gel glass because the CDs achieved complete dispersion in the gel glass to inhibit their aggregation and movement. A low loading ( $<5 \mathrm{wt} \%)$ of CDs resulted in a high solid-state QY (48-88\%), which reached up to $88 \%$ with a CD loading mass ratio of $0.01 \mathrm{wt} \%$ (Fig. 3b). However, a high loading ( $>5 \mathrm{wt} \%)$ caused a low solid-state QY. Zhou et al. ${ }^{53}$ prepared CDs with green emission using CA and urea as raw materials via a one-step microwave method, and then successively added tetraethoxysilane and ammonia water to a $\mathrm{CD}$ ethanol solution to form a $\mathrm{CD} @ \mathrm{SiO}_{2}$ composite gel and realize solid-state fluorescence in the CDs. When the concentration of CDs in the ethanol solution increased from 0.05 to $0.4 \mathrm{mg} \mathrm{mL}^{-1}$, that is, the loading mass ratio of CDs increased from 2.1 to $8.4 \mathrm{wt} \%$, the solid-state QY of the $\mathrm{CD} @ \mathrm{SiO}_{2}$ gel decreased from $52 \%$ to $31 \%$ (Fig. 3c), which was caused by the aggregation and collision of the CDs. However, when the loading mass ratio of CDs increased from 8.4 to 19.2 wt\% with the CD concentration in ethanol solution increasing from 0.4 to $0.8 \mathrm{mg} \mathrm{mL}^{-1}$, their solid-state QY increased from $31 \%$ to $41 \%$ because the formation of a crosslinking structure in CDs can inhibit the aggregation of CDs. The synchronized realization of high solid-state QY and high loading of CDs facilitates the high brightness of the $\mathrm{CD} @ \mathrm{SiO}_{2}$ composite gel.

\subsection{Doping heteroatoms}

The introduction of heteroatoms influences the overall electron distribution and related electron energy levels of CDs. Doping heteroatoms have been proved to be an effective method to regulate the fluorescence and other properties of CDs. Thus far, various heteroatoms, including nitrogen, sulfur, boron, phosphorus, silicon and halogens, have been doped into CDs to improve their performance. Further, heteroatoms co-doping is widely used to promote the doping efficiency, charge distribution and enhance the optical properties of CDs via the synergistic coupling effect between heteroatoms.

Tao et $a l .{ }^{54}$ demonstrated the effect of nitrogen doping on the solid-state fluorescence characteristics of CDs. They synthesized three CDs via a one-step hydrothermal method using polyacrylic acid as the carbon source and three raw materials (ethylenediamine, ethanolamine and ethylene glycol) with different nitrogen contents as additives. The three CDs exhibited solid-state fluorescence characteristics because of the formation of a crosslinked polymer structure. The QYs of the three CDs in solution state was $32.41 \%, 12.29 \%$, and $2.61 \%$, and those in the powder state was $28.77 \%, 11.17 \%$, and $2.13 \%$, respectively. 
Therefore, nitrogen doping plays an important role in the improvement of both the solution and solid-state fluorescence of CDs, and a high nitrogen doping content can cause a high fluorescence emission in CDs.

Choi et al. $^{42}$ synthesized blue emissive N,B-co-doped CDs (N,B-CDs) with a QY of $80.8 \%$ in solution and up to $67.6 \%$ in powder via a one-step microwave method. For comparison, CA was used as the carbon source and ethylenediamine as the nitrogen dopant without boric acid to prepare CDs, which also showed blue emission with a QY of $40.2 \%$ in solution, but only $1 \%$ in powder (Fig. 4a), confirming that the introduction of $\mathrm{B}$ is beneficial to enhance the solid-state luminescence of CDs. This is because B doping can change the bonding ways between $\mathrm{C}$ and $\mathrm{N}$ in CDs, promote the formation of graphitic $\mathrm{N}$, and generate well-distributed surface defect states, leading to an improvement in QY (Fig. 4b). Zheng et al. ${ }^{34}$ prepared blueemitting N,Si-co-doped CDs (N,Si-CDs) with a solid-state QY of 65.8\% (Fig. 4c) using CA and $\mathrm{KH}-792$ as raw materials via a one-step microwave-assisted hydrothermal method. In addition, N,Si-CDs dropped on a glass substrate also showed significant blue fluorescence emission (Fig. 4d). The long chain structure of KH-792 on the surface of N,Si-CDs enabled them to maintain a good dispersion state in powder or film, which caused N,Si-CDs to exhibit bright solid-state luminescence (Fig. 4e).

\subsection{Adding salt crystals in situ and reducing carbonization degree}

Besides the "heteroatom doping" strategy, adding salt crystals in situ or reducing the carbonation degree of reactants are also effective methods for the "one-step" synthesis of high efficiency solid-state fluorescent CDs. On the one hand, the addition of salt crystals, such as $\mathrm{KCl}$ and $\mathrm{NaOH}$, can act as a dispersion matrix to isolate $\mathrm{CD}$ nanoparticles in situ and reduce the formation of defects, both of which contribute to the improvement of the solid-state fluorescence intensity of CDs. On the other hand, controlling the proportion of reactants or energy of the reaction system can avoid the excessive carbonization of the reactants, and thus the CDs have a low conjugated structure, which also contributes to the improvement of the solid-state fluorescence intensity of CDs.

Zhang et $a .^{47}$ prepared two types of CDs via a one-step microwave method using CA and L-cysteine as raw materials with and without $\mathrm{KCl}$, which were named CDs1 and CDs2, respectively. CDs1 exhibited bright solid-state fluorescence with a QY of 65\%, while CDs2 showed no solid-state fluorescence (Fig. 5a and b, respectively). $\mathrm{KCl}$ can act as a temperature conduction medium, and thus the raw materials can be uniformly heated. $\mathrm{KCl}$ can also act as a matrix to separate adjacent nanoparticles and avoid their aggregation (Fig. 5c), causing CDs1 to have a uniform band gap, which can reduce the channels of non-radiation transition, and achieve high solidstate fluorescence. Wei et al. ${ }^{55}$ prepared green fluorescent CDs with a solid-state QY of 75.9\% using CA, urea, and $\mathrm{NaOH}$ as precursors, which is the highest QY of green emissive CDs reported to date. The effect of different amounts of $\mathrm{NaOH}$ on the solid-state luminescence of CDs was studied (Fig. 5d). When $\mathrm{NaOH}$ was added in an appropriate amount, the solidstate fluorescence of the CDs was 472 times higher than that of the CDs without $\mathrm{NaOH}$, which not only resulted in CDs with high crystallinity (Fig. 5e and f) and avoided the formation of defects in the CDs, but also in situ dispersed the CDs to form a large steric hindrance and avoid non-radiative energy transfer between the nanoparticles. This confirmed that $\mathrm{NaOH}$ significantly contributed to the strong solid-state PL intensity of the CDs.

The carbonization degree of CDs can also affect their solidstate luminescence. Yoo et al. ${ }^{56}$ regulated the solid-state QY of CDs by controlling the molar ratio of CA to urea and reaction time to further adjust the $\mathrm{sp}^{2}$ region of the CDs. Specifically, excess CA and urea were used to prepare c-CDs and u-CDs, respectively.
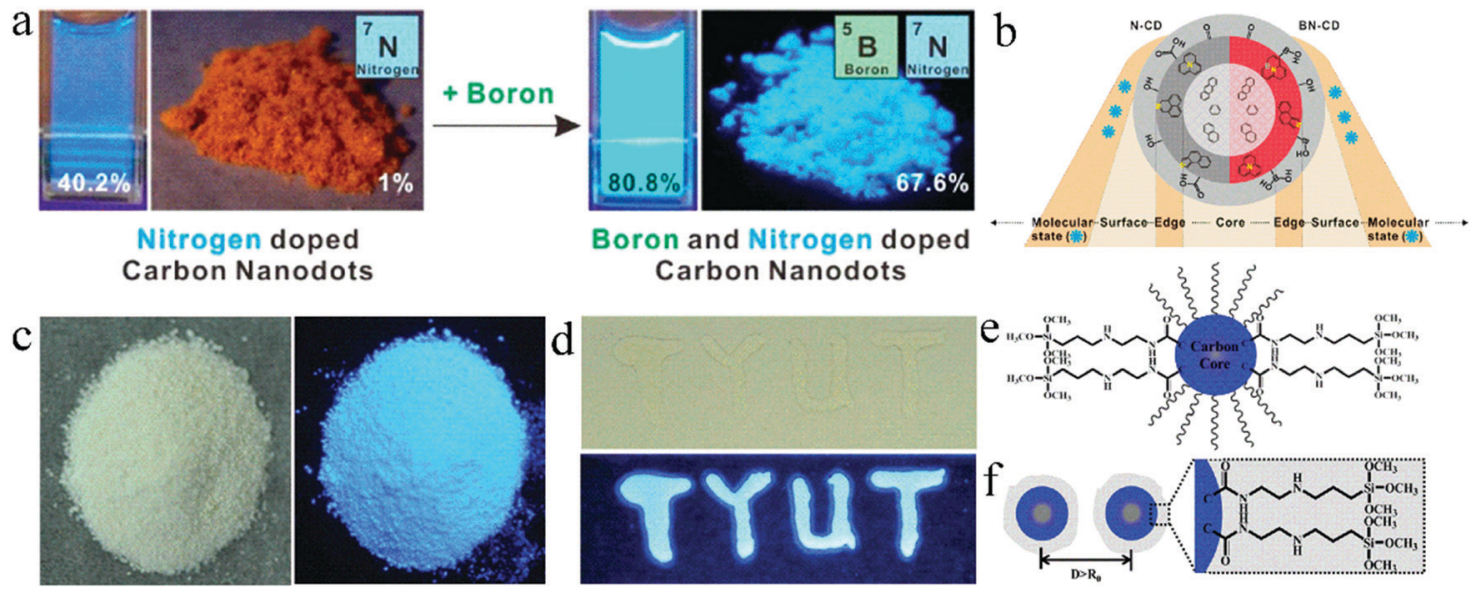

Fig. 4 (a) Photographs of N-doped CDs and N,B-CDs in solution and powder under UV light. (b) Schematic structures of the corresponding CDs. Reproduced with permission from ref. 42. Copyright 2016, the American Chemical Society. (c) Photographs of N,Si-CD powder under daylight (left) and UV light (365 nm) (right). (d) Optical photograph of N,Si-CD films on a glass under daylight (up) and UV light (bottom). (e) Illustration of N,Si-CDs. (f) Schematic of solid-state N,Si-CDs resistant against the ACQ effect. Reproduced with permission from ref. 34. Copyright 2017, The Royal Society of Chemistry 

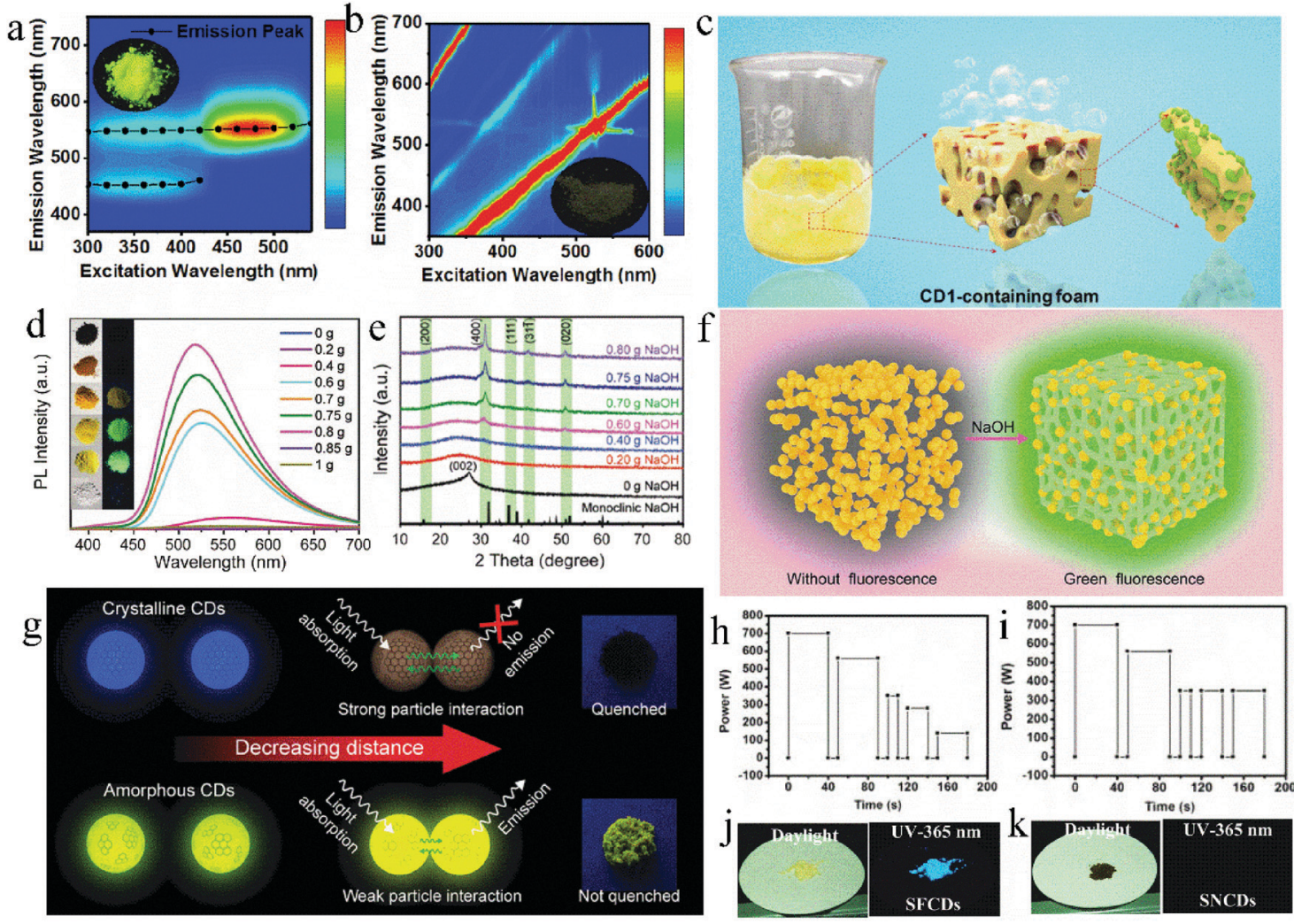

Fig. 5 Excitation-emission matrix of solid CD1 powder (a) and CD2 powder (b), and the inset is the corresponding fluorescent photograph. (c) Schematic of CDs. Reproduced with permission from ref. 47. Copyright 2019, the American Chemical Society. (d) PL spectra of the CDs with different amounts of $\mathrm{NaOH}$. The inset is the comparison photos of the series of samples under natural light and a UV lamp. (e) XRD patterns of the CDs with different amounts of $\mathrm{NaOH}$. (f) Schematic diagram of the solid-state emission process of CDs. Reproduced with permission from ref. 55 . Copyright 2020, John Wiley and Sons. (g) Luminescence quenching schematic of crystalline and amorphous CDs depending on the amount of sp ${ }^{2}$ domain. Reproduced with permission from ref. 56. Copyright 2019, the American Chemical Society. Procedure for the microwave synthesis of (h) SFCDs and (i) SNCDs. Photographs of (j) SFCD and (k) SNCD powder under daylight and a UV-lamp. Reproduced with permission from ref. 57. Copyright 2016, The Royal Society of Chemistry.

The former suffered from serious solid-state fluorescence quenching with a QY of $0 \%$, but the latter exhibited yellow solid-state fluorescence emission centered at $530 \mathrm{~nm}$ with a QY of $1.3 \%$. This is because the $\mathrm{N}$, O-containing functional groups in urea hindered the growth of the $\mathrm{sp}^{2}$ region, reduced the $\pi-\pi$ stacking among CDs, and finally inhibited the solid-state quenching of u-CDs. To verify this hypothesis, the synthesis time of c-CDs was shortened from 5 to $3 \mathrm{~min}$. Consequently, the carbonization degree of the CDs was reduced and the $\mathrm{sp}^{2}$ region was reduced, thus the solidstate QY of c-CDs increased from $0 \%$ to $7.8 \%$. At a close distance (Fig. 5g), the crystalline CDs and amorphous CDs exhibited different behaviors originating from their different amounts of $\mathrm{sp}^{2}$ domain. The extended $\mathrm{sp}^{2}$ conjugation system in the crystalline CDs intensified the CD solid-state quenching. Therefore, reducing the $\mathrm{sp}^{2}$ region in CDs can enhance their solid-state luminescence. Zhang et $a .^{57}$ synthesized solid-state fluorescent CDs (SFCDs) and solid-state non-fluorescent CDs (SNCDs) using $\mathrm{CA}$ and urea under different microwave reaction conditions (Fig. $5 \mathrm{~h}$ and i, respectively). As shown in Fig. 5h, the reduced power after the third stage was to avoid sharp heating and excessive carbonation, while the reaction power was not reduced in Fig. 5i. The solid-state QY of SFCDs and SNCDs was $40 \%$ and $1 \%$ (Fig. $5 \mathrm{j}$ and $\mathrm{k}$ ), respectively, which is because SNCDs experienced more serious dehydration and carbonization, resulting in luminescence quenching.

\section{The methods for multicolor tuning of solid-state fluorescent CDs}

Multicolor-adjustable fluorescent materials exhibit extensive application potential in the fields of luminescent display and lighting. Researchers have achieved multicolor-adjustable solidstate fluorescence in CDs by controlling their aggregated state or luminescence center. The main methods can be summarized as follows: effective dispersion of multicolor fluorescent CDs, gradient dispersion of concentration-induced multicolor-emissive CDs, and regulation of fluorescence center of self-quenching-resistant CDs. We will discuss each of these in the following sections.

\subsection{Dispersing multicolor CDs}

Multicolor fluorescent CDs are dispersed in a matrix to keep the luminescence emission consistent between the solid state and liquid state, thus achieving the preparation of multicolor 
solid-state fluorescent CDs. Wang et al. ${ }^{58}$ adsorbed blue emissive CDs (B-CDs) and orange-emissive CDs (O-CDs) on PS through self-assembly technology, and synthesized a solid luminescent CD@PS composite phosphor (Fig. 6a). Further, by adjusting the mass ratio of B-CDs to O-CDs, CD@PS composite phosphors with polychromatic luminescence were synthesized. When the mass ratio of B-CDs to O-CDs decreased from $2: 1$ to $0.8: 1$, the luminescence of the CD@PS composite phosphors changed from orange to blue, and centered at the white region at the mass ratio of $1.2: 1$ (Fig. $6 \mathrm{~b}$ and c). Similarly, Bhunia et al. ${ }^{59}$ mixed a variety of CDs and polydimethylsiloxane (PDMS) to prepare multicolor CD@PDMS, where the CDs were in situ embedded in PDMS. When D-glucose, L-ascorbic acid, and vitamin B1 were used as the carbon sources, the CD@PDMS film exhibited bright green, yellow and orange emission, respectively. When D-glucose and L-ascorbic acid were mixed as precursors with a mass ratio of $3: 1$, the CD@PDMS film produced white fluorescence (Fig. 6d).
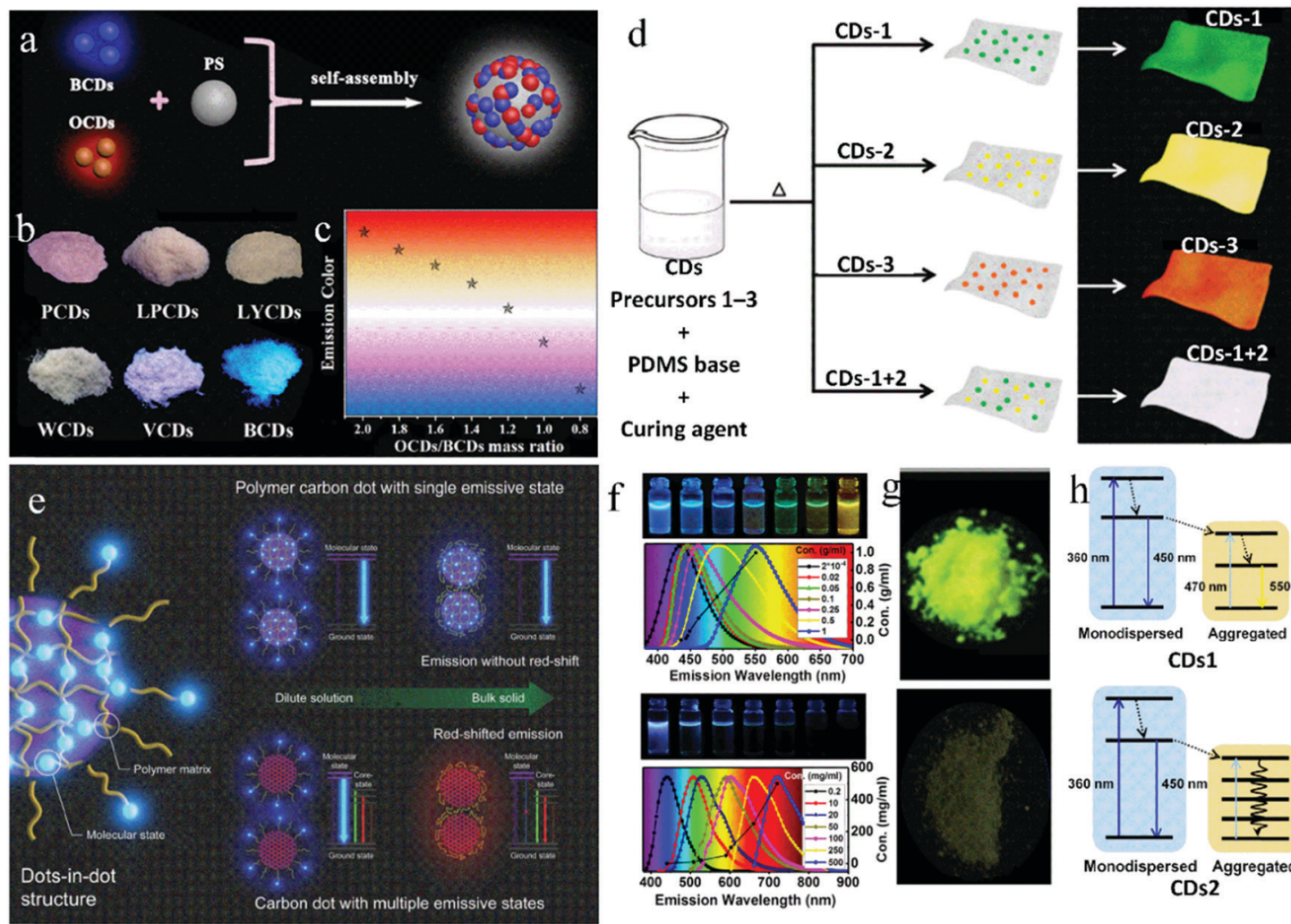

f
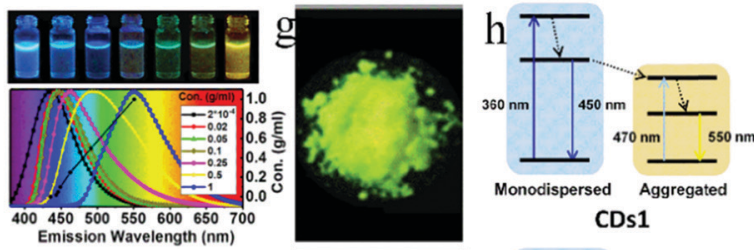

CDs1
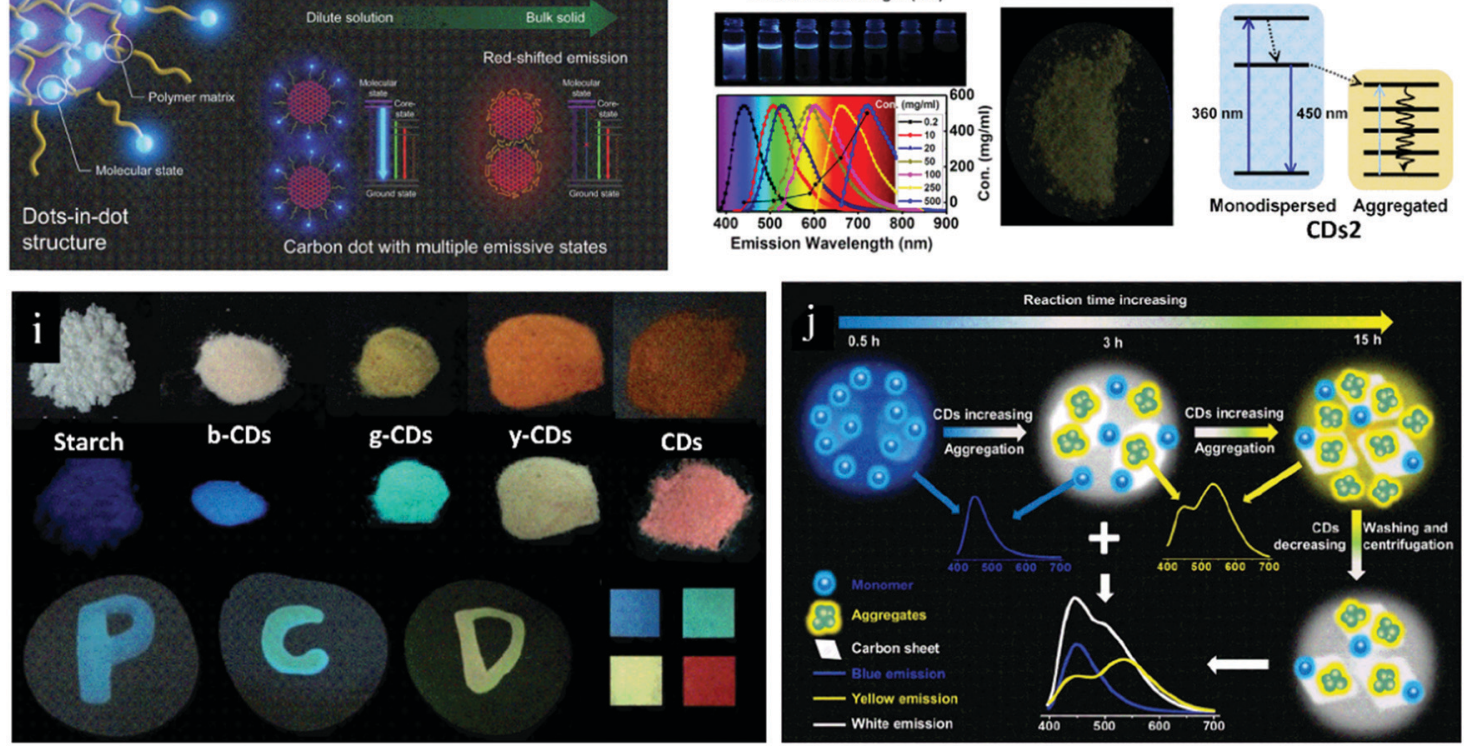

Fig. 6 (a) Schematic illustration of the assembly of white CD@PS nanocomposites. (b) Fluorescence photographs of a series of CD@PS composites with various mass ratios of O-CDs and B-CDs. (c) Emission colors of CD@PS composites. Reproduced with permission from ref. 58. Copyright 2019, the American Chemical Society. (d) Scheme depicting the preparation of the mixed films via a one-pot thermal synthetic route. Reproduced with permission from ref. 59. Copyright 2016, The Royal Society of Chemistry. (e) Schematic illustration of PDs and their mechanism for solid-state luminescence with emission color consistency compared to CDs with multiple emissive states. Reproduced with permission from ref. 66. Copyright 2019, John Wiley and Sons. (f) Fluorescent photographs of CD1 (top) and CD2 (down) solutions at different concentrations and corresponding normalized PL spectra. (g) Fluorescent photographs of solid-state CD1 (top) and CD2 (down). (h) Schematic of energy transfer occurring in the aggregation process of CDs1 (top) and CDs2 (down). Reproduced with permission from ref. 47. Copyright 2019, the American Chemical Society. (i) Nanocomposite and print ink applications of PDs. Reproduced with permission from ref. 68. Copyright 2017, John Wiley and Sons. (j) Schematic of the construction of multicolor CD/ nanosheet hybrid emitters. Reproduced with permission from ref. 70. Copyright 2019, The Royal Society of Chemistry. 


\subsection{Dispersing concentration-induced multicolor CDs with gradient concentration}

The concentration-dependent property of CDs is that CDs emit different fluorescence at different concentrations, which often manifests itself as a redshift in luminescence with an increase in solution concentration, that is, concentration-induced luminescence redshift. By combining the concentration-induced luminescence redshift characteristics of CDs and the strategy of quenching resistance, multicolor solid-state luminescence of CDs can be realized. The concentration-induced luminescence redshift of CDs can be realized by either dispersing them in a solid matrix with a gradient concentration through a postprocess, or in situ embedding in a matrix with a gradient concentration by controlling the process parameters.

Some researchers believe that the concentration-induced luminescence redshift of CDs is caused by the formation of aggregated states at high concentrations. Song et al. ${ }^{60}$ obtained CDs by refluxing concentrated mixed acid, and their emissive wavelength changed gradually from green to red with an increase in their concentration. The redshift mainly depends on the self-assembly of the CDs under different concentrations, namely, in dilute solution, the emission is mainly from monodisperse CDs, while in high concentrations, CDs assemble into aggregates through hydrogen bonding, and the energy transfer between aggregates occurs and induces a long wavelength emission. Lai et $a{ }^{61}{ }^{61}$ reported a similar study. They synthesized graphene CDs with concentration-induced redshift properties by cutting oxidized graphene in $\mathrm{H}_{2} \mathrm{O}_{2}$. They proposed that aggregation occurs with an increase in solution concentration, causing a large $\mathrm{sp}^{2}$ conjugated region and low energy gap, which will lead to a red shift in emission. Meng et al. ${ }^{62}$ synthesized CDs with concentration-dependent characteristics and solid-state red fluorescence from a mixed solution of coal pitch powder, formic acid and $\mathrm{H}_{2} \mathrm{O}_{2}$ as the precursors. As the concentration of CD solution increased from 0.03 to $3 \mathrm{mg} \mathrm{mL}$ until it became powder, the luminescence wavelength shifted from 430 to $630 \mathrm{~nm}$. They attributed this phenomenon to the decrease in the band gap of the CDs. Ba et al. ${ }^{63}$ prepared CDs with concentration-dependent properties and yellow solid-state fluorescence after the hydrothermal carbonization of CA and ammonium hydroxide, and further illuminated the mechanism of these properties. In the dispersed state, blue emission mainly comes from the transition in the $\mathrm{sp}^{2}$ graphite region. At high concentration, the distance between the CD nanoparticles deceases, which induces charge redistribution, and the surface state with a narrower band gap becomes the dominant emission source, causing green emission. While, in the solid state, a large number of mono-dispersed CDs regularly aggregate, and the $\mathrm{sp}^{2}$ conjugated region expands and the band gap becomes narrower, causing yellow light emission.

Other researchers believe that the concentration-induced luminescence redshift of CDs is caused by multiple emission centers. Chen et al. ${ }^{64}$ synthesized CDs with three emission centers containing a carbon core, edge state, and surface state via a onestep hydrothermal method using CA and ethanolamine as raw materials. At high concentration, the distance between multiple emission centers decreased and energy transfer occurred, which led to a concentration-induced luminescence redshift.

Yan et al. ${ }^{65}$ synthesized CDs with CA and 1-(2-pyridine azo)2-naphthol via the hydrothermal method, which showed three emission centers located at 375, 450 and $595 \mathrm{~nm}$. With an increase in concentration, the emission at $595 \mathrm{~nm}$ gradually increased, while the emission at $375 \mathrm{~nm}$ and $450 \mathrm{~nm}$ decreased, showing an emission redshift. This is because as the concentration increases, the distance between the emission centers decreases, and energy transfer occurs from the short-wavelength emissive components to the long-wavelength components. Kwak et $a l .{ }^{66}$ proposed a similar view, that is, the existence of multiple emission centers in CDs is beneficial for the concentrationinduced luminescence redshift. On the contrary, the existence of a single emission center is conducive to the color consistency of CDs at different concentrations. Blue-emitting PDs were synthesized via the heat treatment of CA and diethylenetriamine at $70{ }^{\circ} \mathrm{C}$, whose QY in solid state $(62.7 \%)$ was similar to that in solution $(68.2 \%)$. The luminescence maximum of the PDs was located at $434 \mathrm{~nm}$, and the fluorescence originated from the molecular state. In PDs, the excessive amide bonds and oligomers acted as dispersion media, and thus the fluorophores were uniformly dispersed through cross-linking. This special structure can avoid direct contact between fluorophores and kept the fluorescence color consistent for the PDs in the solution and powder state. With a higher reaction temperature $\left(200{ }^{\circ} \mathrm{C}\right)$ and the same raw materials, PDs with significant concentrationdependent characteristics were prepared. After a detailed structural analysis, it was found that at high temperature, the polymer structure was destroyed, and an $\mathrm{sp}^{2}$ region with crystal structure was formed; thus, the molecular state and carbon core state together caused the fluorescence emission in the CDs. It can be seen that the existence of multiple emission states in the CDs contributes to the concentration redshift. While, the single emission state in PDs is the main reason for their consistent luminescence (Fig. 6e).

Not all CDs with a concentration-induced redshift emit PL in the solid state. In fact, some of them show solid-state fluorescence, ${ }^{67}$ while others experience solid-state quenching. ${ }^{47}$ Zhang et $a .^{47}$ first explored this phenomenon in detail. With and without $\mathrm{KCl}$, CDs1 and CDs2 were synthesized, respectively, via a one-step microwave method with $\mathrm{CA}$ and L-cysteine as raw materials. The dilute solution of both showed blue emission and the property of concentration-induced luminescence redshift (Fig. 6f). The difference was that the CDs1 powder showed bright yellow fluorescence, while CDs2 powder showed almost no fluorescence (Fig. 6g). This is because monodispersed CDs1 aggregated as their concentration increased, and the residual dispersed CDs1 with blue emission transmitted their energy to the aggregates, giving them yellow light emission (Fig. 6h). However, for CDs2, because of the strong and wide absorption of the CDs2 aggregates, the energy of the residual dispersed CDs2 was dissipated through the non-radiative transition of the aggregates, thus inducing fluorescence quenching (Fig. 6h). In this case, $\mathrm{KCl}$ acted as a temperature conduction medium, which enabled the raw materials to be uniformly heated, CDs1 isolated, 
and overgrowth avoided. Consequently, CDs1 had a uniform particle size, which avoided the non-radiation energy dissipation in the aggregated state, and thus achieved solid-state luminescence.

By utilizing the significant concentration-dependent characteristics of CDs, Shao et al. ${ }^{68}$ realized multicolor solid-state luminescent PDs. The PDs were prepared via a one-step microwave method with maleic acid and EDA as raw materials. By controlling the microwave conditions and further carbonization of the raw materials, the emission of PDs was realized in the red region with the solid-state QY of $8.5 \%$. By dispersing in water or other solid matrices at different concentrations, the PDs formed different degrees of supramolecular crosslinking, thus inducing different PL colors. Taking advantage of their unique concentration-dependent property, multicolor solid CD-based composites from blue to red were prepared by changing the proportion of PDs in the starch matrix (Fig. 6i).

In the above reports, multicolor CDs were obtained after their dispersion in a solid matrix in varying proportions. Different from the above reports, in other studies, precursors with unique structures were used to form the dispersion matrix through hydrolysis or self-assembly to effectively in situ disperse CDs. Accordingly, multicolor solid luminescent CDs can be obtained.

Zhan et $a l .{ }^{69}$ realized the solid-state emission of CDs through their dispersion in $\mathrm{SiO}_{2}$, and then obtained multicolor solid-state fluorescence CDs through gradient concentration dispersion in $\mathrm{SiO}_{2}$. Here, CA and (aminopropyl)triethoxysilane (APTES) were used as the raw materials to prepare CDs, where APTES was hydrolyzed to form $\mathrm{SiO}_{2}$ to in situ disperse the CDs. By adjusting the proportion of raw materials and mixed solvent (water and ethanol), the CDs were dispersed in $\mathrm{SiO}_{2}$ in different proportions. Consequently, the fluorescence emission of the CDs exhibited different redshifts, that is, a higher loading of $\mathrm{CDs}$ in $\mathrm{SiO}_{2}$ led to a more significant redshift in luminescence because of the greater FRET and reabsorption. Among them, the solid-state QY of the blue-, yellow- and orange-emissive CDs was up to $57.9 \%, 34.3 \%$, and $32.7 \%$, respectively. Chang et al. ${ }^{70}$ regulated the proportion of CDs in self-assembled nanosheets by changing the reaction time, thus in situ dispersing CDs with different concentrations to realize multicolor luminescence. By using CA and octadecylamine as the raw materials and octadecene as the solvent, CDs were synthesized via the solvothermal method. Here, octadecylamine not only participates in the formation of CDs as a reaction precursor, making the CDs passivated, but also self-assembled into nanosheets as a dispersion matrix to in situ disperse the CDs and construct a CD/ nanosheet complex. Consequently, the solid fluorescence of the CDs was realized. When the reaction time was short $(0.5 \mathrm{~h})$, the CDs were effectively dispersed in the nanosheets, realizing blue emission. With an extension of the reaction time, the degree of dehydrogenation and carbonization increased, and the CDs had greater aggregation, leading to a stronger $\pi-\pi$ interaction and FRET, thus giving a yellow emission (Fig. 6j). Similar with the above work, the research by Cheng et al. ${ }^{71}$ showed that a $\mathrm{CD} /$ micro-belt hybrid was prepared via the solvothermal method with oxalic acid and cetylamine as raw materials, and oleic as the solvent. A differential washing method was adopted to realize the multicolor emission of the $\mathrm{CD} / \mathrm{micro}$-belt hybrid. Specifically, with an extension the washing time, the CD aggregation decreased, causing a blue shift in the emission. The solid-state QY of the white-, green-, and blue-emissive CD/ micro-belt hybrid was $32 \%, 35 \%$, and $34 \%$, respectively.

\subsection{Tuning fluorescence origin of self-quenching-resistant CDs}

The fluorescence of CDs originates from their carbon core, surface state or molecular state. Their different luminescence centers usually show obvious differences in the fluorescence spectrum. Therefore, a change in the particle size and surface functional groups, and the conversion of the luminescent centers can cause a shift in the emission peak of CDs.

Most studies have shown that CDs with a large particle size $\left(\mathrm{sp}^{2}\right.$ conjugated degree or high graphitization degree), high contents of $-\mathrm{COOH}$, and high nitrogen doping ratio can solely or synergistically cause a redshift in the emission wavelength of CDs. Also, by regulating the degree of crosslinking and carbonization of the raw materials, the fluorescence center of CDs can be tuned to realize the regulation of their fluorescence color. For self-quenching-resistant CDs, multi-color solid-state luminescence can be realized when their particle size and surface functional groups are changed or luminous centers are transformed by controlling the raw materials, reaction temperature, reaction time, $\mathrm{pH}$, and other parameters.

In some research, multicolor emissive CDs can be obtained by regulating the graphitization degree of CDs. Shen et al. ${ }^{72}$ synthesized multicolor CDs by changing the reaction solvent. With sodium citrate dihydrate and urea as raw materials, CDs were prepared via the solvothermal method, during which, the CDs were in situ embedded into sodium citrate crystals, showing solid-state emission. When the reaction solvents were diethylformamide, dimethylacetamide, and DMF, the emission wavelength of the solid-state CDs was centered at 513, 530 and $537 \mathrm{~nm}$, and the solid-state QY was $21.6 \%, 18.7 \%$ and $18.6 \%$, respectively. DMF can promote the carbonization of raw materials, leading to CDs with a high graphitization degree and long-wavelength emission.

In other reports, multicolor emissive CDs were obtained by regulating their surface states. By controlling the mass ratio of reactants, Wang et al. ${ }^{16}$ synthesized multicolor CDs from CA and piperazine as raw materials via a microwave method. When the mass ratio of CA to piperazine was $1: 0.5,1: 1$, and $1: 2$, the optimal emission of the CD powder was located at 436, 503, and $536 \mathrm{~nm}$, respectively. The detailed structural analysis showed that an increase of the emission wavelength gradually increased with the content of amide $\mathrm{C}=\mathrm{O}$ in the CDs. It can be seen that the difference in the luminescence wavelength was caused by the different surface defect states.

By changing the amount of nitric acid to regulate the $-\mathrm{COOH}$ content on the surface of CDs, Song et al. ${ }^{73}$ prepared multicolor solid-state CDs from waste plastic (PS as the main ingredient) through a one-step solvothermal method, where the long chain structure of PS played a key role in suppressing the 
solid-state quenching of the CDs. When addition amount of nitric acid increased from 5 to 10 up to $30 \mu \mathrm{L}$, the emission of the solid-state CDs changed from white to yellow to orange, which were named W-, Y-, and O-CDs with a solid QY of 5.2\%, $3.4 \%$ and $3.1 \%$, respectively (Fig. $7 \mathrm{a}$ ). This is because with an increase in the dosage of nitric acid, the oxygen content on the surface of the CDs increased from 3.83\% to $6.02 \%$ (Fig. 7b), and consequently, the increase in oxidation degree led to a decrease in the band gap and redshift of the emission wavelength of the CDs.

The graphitization degree and surface state of CDs can also collaboratively regulate their luminescence wavelength. Yang et $a .^{74}$ synthesized CDs via the reaction of CA and thiourea in acetone, and in situ embedded CDs in a sandwich manner in a two-dimensional laminated matrix, which increased the distance between the $\mathrm{CD}$ nanoparticles, making them evenly dispersed and avoiding fluorescence quenching. Furthermore, with an increase in the thiourea content, reaction temperature, and reaction time, the degree of graphitization and content of - $\mathrm{COOH}$ gradually increased. Consequently, the luminescence wavelength of the CDs gradually shifted from 410 to $610 \mathrm{~nm}$. Zhu et al. ${ }^{75}$ developed a seed growth strategy to prepare multicolor luminescent solid-state CDs by changing the amount of seed CDs and reaction time to regulate the carbon core size and nitrogen doping content (Fig. 7c). Here, the CDs were re-grown through the hydrothermal reaction with CA and urea as seed CDs, PVP and urea as additives, and DMF as the solvent. In this case, PVP played an important role in suppressing the solidstate quenching of the CDs. The reaction conditions and schematic representation of the four typical CDs are shown in Fig. 7d. The sizes of the four CDs were 1.6, 3.6, 5.0 and $5.6 \mathrm{~nm}$, and the nitrogen contents were $4.7 \%, 11.6 \%, 15.3 \%$ and $24.6 \%$, respectively, where both the changes in the particle size and nitrogen content caused a shift in the emission wavelength from 425 to $580 \mathrm{~nm}$ with a solid-state QY of $17-26 \%$. The red shift in the luminescence wavelength is mainly caused by the increase in the CD particle size, and also by the increase in the nitrogen doping content.

Multi-color luminescence in CDs can also be achieved by the transformation of their luminescence center. By controlling the reaction temperature, Zhou et al. ${ }^{76}$ realized the transformation of the luminescence center of CDs. Specifically, with an increase in the reaction temperature, the luminescence center of the CDs changed from the molecular state to carbon core state, and accordingly the solid-state fluorescence of the CDs changed from blue to green. In the presence of $\mathrm{CaCl}_{2}$, CDs were synthesized with CA and urea by vacuum heating. The volatile $\mathrm{HCl}$ generated in the reaction process was pumped out, and the
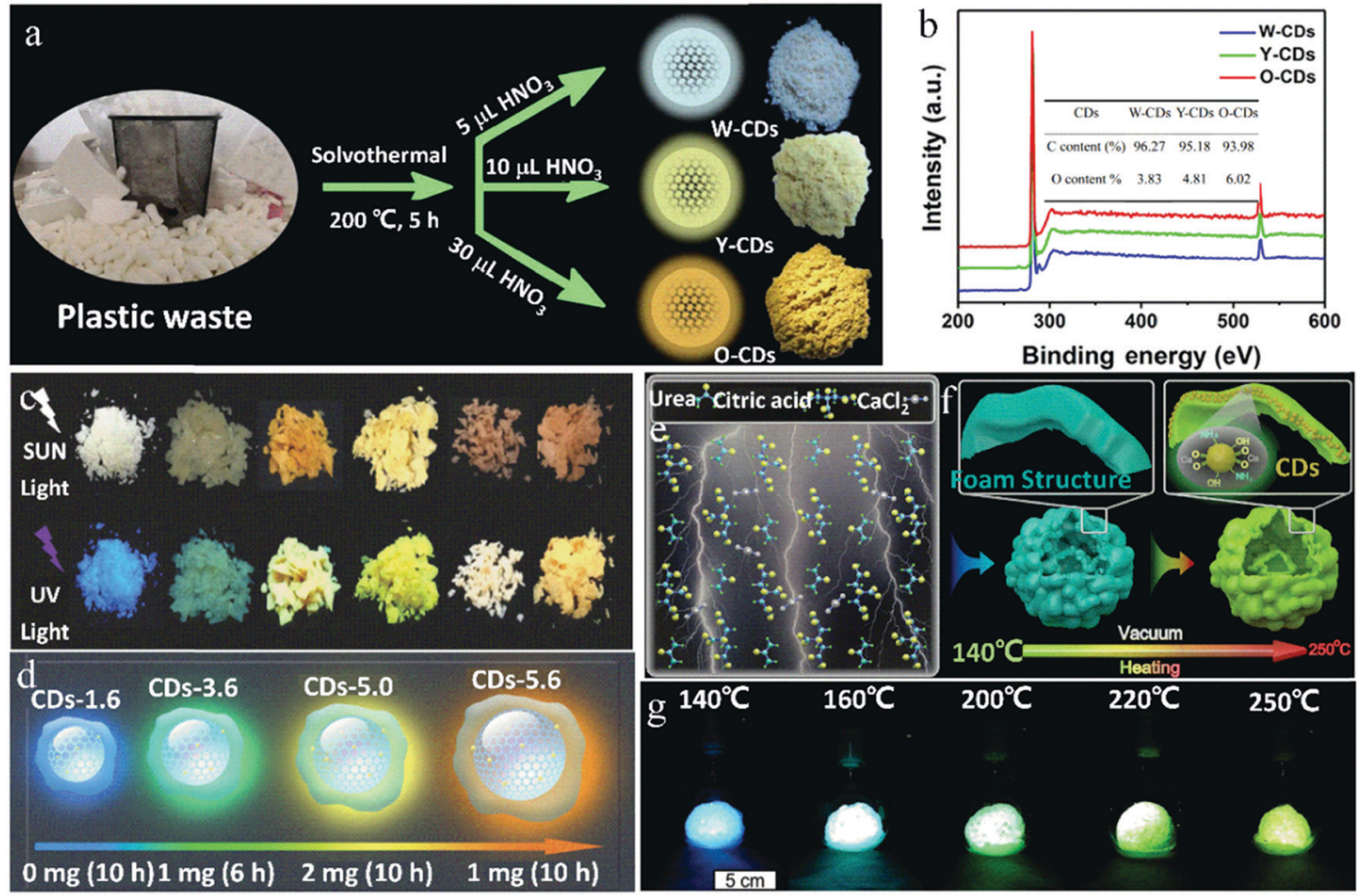

Fig. 7 (a) Schematic of the fabrication and (b) full XPS spectra of solid-state fluorescent W-CDs, Y-CDs and O-CDs. Inset of (b) is the contents of carbon and oxygen of CDs. Reproduced with permission from ref. 73. Copyright 2019, Elsevier. (c) Photographs of the six CD powders (from left to right: CD-1.6, CD-3.6, CD-4.6, D-5.0, CD-5.4, and CD-5.6) under sunlight and UV light. (d) Schematic representation of four different selected sizes and the different extent of $\pi$-conjugated domains of the resulting CDs. Reproduced with permission from ref. 75. Copyright 2019, John Wiley and Sons. (e and f) Schematic of the growth mechanism of CDs synthesized by the vacuum heating method. (g) Photographs of CDs with an inflated foam structure prepared by a vacuum heating method at different temperatures, taken under UV light. Reproduced with permission from ref. 76. Copyright 2019, The Royal Society of Chemistry. 
product formed a foam structure. In addition, calcium carboxylate acted as a medium to isolate the luminescence center, giving the CDs solid-state fluorescence characteristics (Fig. 7e). Further, the CDs showed blue emission when reacted at $140-160{ }^{\circ} \mathrm{C}$, while green emission when reacted at $220-250{ }^{\circ} \mathrm{C}$. At a low temperature (140-160 ${ }^{\circ} \mathrm{C}$ ), the amino-related fluorescent molecules were the main source of luminescence, and the CDs showed blue emission. However, at high temperature $\left(250{ }^{\circ} \mathrm{C}\right)$, the fluorescent molecules were further dehydrated and carbonized into the carbon core state. Here, the carbon core state became the main luminescence center and the CDs showed green emission (Fig. 7f and g). Therefore, multicolor CDs can be realized by controlling the reaction temperature and further adjusting the emission origin of the CDs.

\section{The application of solid-state fluorescent CDs}

Compared with traditional semiconductor quantum dots, CDs have the advantages of low toxicity, easy functionalization and mild reaction conditions. In addition, CDs exhibit the advantages of diversified raw material sources, simple preparation, good chemical stability and light bleaching resistance, and thus are superior to organic fluorescent dyes. Combined with their excellent optical properties, such as adjustable excitation and emission wavelength range, CDs show wide application prospect in many fields and attracted extensive attention from researchers. Among them, solid-state fluorescent CDs show great prospects in the field of solid-state luminescent application, including fingerprint identification, ${ }^{77}$ photoelectric devices and visible light communication. ${ }^{5,78}$

\subsection{Fingerprint identification}

Latent fingerprints (LFPs), which are left on the surface of objects after the finger or hand touching objects, are important physical evidence in criminology and individual identification. However, in most cases, LFPs are invisible to the naked eye because of their poor optical contrast. ${ }^{79}$ LFP imaging is one of most important methods to identify individuals. Therefore, developing methods to achieve clear visualization of LFPs has attracted great attention. Presently, LFP imaging is commonly obtained by solution spraying, which suffers from imaging decaying and information loss with aging time. In contrast, the powder method, although in its infancy, not only can realize the clear display of LFPs, but also maintain permanent stability, suggesting its broad application prospect in fingerprint identification. Because of the excellent photoluminescence properties, good biocompatibility, and simple preparation of CDs, they have a promising future in the field of LFPs. Moreover, the sensitivity and selectivity of LFPs can be improved by the monodispersity and uniform particle size distribution of CDs. The high fluorescence intensity of CDs is helpful for the contrast of LFPs.

Fernandes et al. ${ }^{80}$ brushed $\mathrm{CDs} / \mathrm{SiO}_{2}$ composite powder on different bases containing fingerprints, such as a glass slide, polymer, paper, soft beverage bottle foils and metal surfaces. Taking advantage of the excitation-dependent fluorescence characteristic of CDs, multicolor LFP imaging on different bases was obtained. With CA and glutathione as precursors by a one-step microwave method, Wang et al. ${ }^{81}$ synthesized CDs with bright fluorescence in both the solution and solid state. Then, LFPs were obtained on different substrates by the solution or powder method (Fig. 8a). When the solution method was adopted, the obtained LFPs on the porous substrate showed a bright fluorescent pattern, where the fingerprint details could be clearly identified. However, the LFPs deposited on porous substrates (ceramic and printing paper) exhibited poor resolution and high background noise. With an extension of the aging time, some details of the substructures were lost. However, when the powder method was used, the penetration of the CDs into the porous matrix structure decreased, and the LFPs of different volunteers on different non-porous or porous substrates could be clearly identified. The details of LFPs were not lost over time, and the substructure of the LFPs could still be detected even 30 days later (Fig. 8b). Therefore, the powder method is obviously superior to the solution method, and solid fluorescent CDs have good prospect for LFP visualization. Bandi et al. ${ }^{82}$ applied CD powder for the rapid visualization of LFPs on various porous and nonporous surfaces (Fig. 8c). By virtue of their green emission, CDs can provide a clear enough contrast even on auto-fluorescent surfaces. The LFPs based on CDs could clearly present level 2 and 3 ridge details, which is sufficient for individual identification.

\subsection{Photoelectric device}

Solid-state luminescent CDs also have a good application prospect in the field of solid-state lighting, including photoelectric devices,

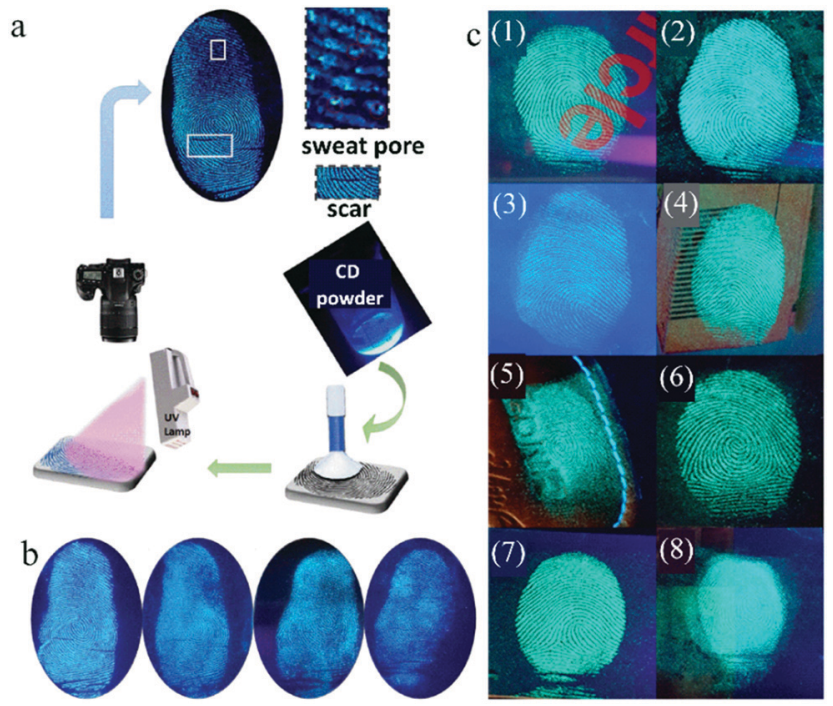

Fig. 8 (a) Scheme of LFP detection through the powder method. (b) Fluorescent images of aged 0 day, aged 7 days, aged 15 days and aged 30 days (from left to right). Reproduced with permission from ref. 81. Copyright 2018, John Wiley and Sons. (c) Fluorescent images of LFPs on different substrate surfaces under $365 \mathrm{~nm}$ UV light: (1) compact disc, (2) glass slide, (3) auto fluorescent lid, (4) magazine paper, (5) leather keychain, (6) floppy disk, (7) aluminum surface, and (8) journal cover page. Reproduced with permission from ref. 82. Copyright 2020, Elsevier. 
Table 1 Summary of CD-based white photoelectric devices

\begin{tabular}{|c|c|c|c|c|c|c|c|c|}
\hline \multicolumn{3}{|c|}{ CDs } & \multicolumn{6}{|c|}{ Photoelectric device } \\
\hline 84 & White & - & Superglue & UV & $(0.34,0.37)$ & - & - & LED \\
\hline \multirow[t]{2}{*}{87} & Red & 16.4 & None & Blue & $(0.33,0.30)$ & $5592-8732$ & 88 & LED \\
\hline & Green & 5.5 & & & & & & \\
\hline \multirow[t]{2}{*}{88} & Blue & 52.6 & Epoxy resin & UV & $(0.33,0.34)$ & 5606 & 92 & LED \\
\hline & Green & 35.1 & & & & & & \\
\hline & Green & 73.0 & & & & & & \\
\hline & Red & 53.0 & & & & & & \\
\hline \multirow[t]{3}{*}{90} & Blue & 57.3 & None & UV & $(0.33,0.33)$ & $3466-7368$ & 88 & LED \\
\hline & Green & - & & & & & & \\
\hline & Red & - & & & & & & \\
\hline 79 & Blue & 60.0 & None & - & $(0.40,0.43)$ & - & 82 & QLED \\
\hline
\end{tabular}

mainly as a phosphor for light-emitting diodes (LEDs), as the light-emitting layer for quantum dot light-emitting diodes (QLEDs), and as a gain medium or phosphor for laser devices (LDs). The next sections will describe each of these.

5.2.1 The application of solid-state fluorescent CDs as phosphors in LEDs. LEDs are core component of lighting and display because of their multiple advantages, such as energy saving, environmental protection, small size, and long life. They have been widely used in various fields, including lighting, display screens and signal lamps.$^{83}$ Solid-state fluorescent CDs can be used as phosphors for LEDs because of their excellent photoluminescence performance. When applied in LEDs, CDs need to be coated on optical lens and to go through curing into a fluorescent film. Therefore, outstanding filmforming ability is important for CDs. Otherwise, they need to be encapsulated in a dispersant to realize curing and film-forming, which makes the fabrication process of LEDs complicated. ${ }^{25}$ In addition, UV and blue chips are usually used to fabricate white LEDs, and thus the excellent absorption of CDs in the UV-blue region is also necessary, which can avoid light leak of the chips.

For the first time, Guo et al. ${ }^{84}$ demonstrated the use of solidstate CDs as a phosphor for LEDs. By controlling the reaction temperature, they prepared CDs with blue, orange, and white fluorescence emission and applied them to LEDs. To avoid the solid PL quenching, the CDs were packaged in superglue and then combined with a UV chip to prepare blue, orange, and white LEDs. The detailed parameters of the CDs and devices are listed in Table 1. Here, the role of superglue was to disperse the CDs and to form a CD film on the lamp cap of the LEDs. Aimed at giving the CDs self-filming property and simplifying the preparation process of LED, Zhang et al. ${ }^{85}$ and Xie et al. ${ }^{86}$ used CA and KH-792 to in situ synthesize blue-emissive CDs, which showed excellent solid-state luminescence, film forming, and thermal- and UV-resistant characteristics. Combined with the UV chip, the CDs were used for the preparation of white LEDs. To avoid poor compatibility between the CDs and packaging materials, Wang et al. ${ }^{87}$ constructed CDs that can directly cross-link and solidify. They used CA and $p$-phenylenediamine as the carbon sources, and AEAPMS and 3-isocyanylpropyltriethoxysilane as the coupling agents to synthesize green- and red-emissive CDs, respectively. Further, these two CDs were combined with a blue light chip to prepare white light LEDs with a CRI of up to 88. Here, two classes of CDs can form a 3D cross-linked structure through the hydrolysis and crosslinking of alkoxysilane, making them self-dispersive and self-filming. In this study, good compatibility between the CDs and packaging materials in the LEDs was realized, and the film-forming property of the phosphors was realized.

It is generally assumed that a blue chip will damage the human eyes. Therefore, in other reports, white LEDs were prepared by combining UV chips and red-, green- and blueemissive CD phosphors. Miao et al. ${ }^{88}$ prepared high-quality white LEDs by using triple-color CDs as phosphors combined with a UV chip. Firstly, multicolor CDs were prepared with $\mathrm{CA}$ and urea as the raw materials by controlling the reaction temperature and the ratio of raw materials to regulate the graphitization degree and - $\mathrm{COOH}$ contents of the CDs. Then, the triple-color CDs were packaged in epoxy resin to obtain $\mathrm{CD}$ films (Fig. 9a and b). Finally, white LEDs were prepared by the combination of three primary CD films and a UV chip (Fig. 9c and d), and these parameters did not change in 20 days of operation, indicating their good stability. To further improve the CRI of LEDs, Wang et al. ${ }^{89}$ synthesized CDs with highefficiency red emission, solving the defect of insufficient red emissive composition in LEDs. Red-emissive CDs with a QY of up to $53 \%$ were synthesized via the solvothermal treatment of 1,3-dihydroxynaphthalene and $\mathrm{KIO}_{4}$. Then, the red-emissive CDs were mixed with blue and green CDs (Fig. 9e and f) in PMMA and combined with a UV chip to prepare LEDs (Fig. 9g and h). The PL intensity of the LEDs maintained $90 \%$ retention of the initial value after $72 \mathrm{~h}$ of operation. Ding et al. ${ }^{90}$ mixed green CDs with red CDs and film-forming blue CDs to make a white fluorescent film with film-forming 
a

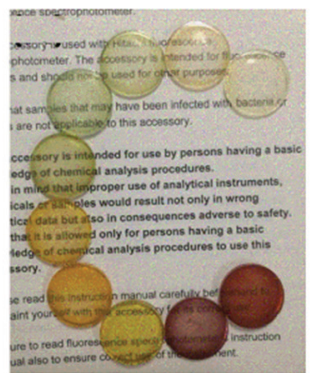

$\mathrm{C}_{\text {s }}$
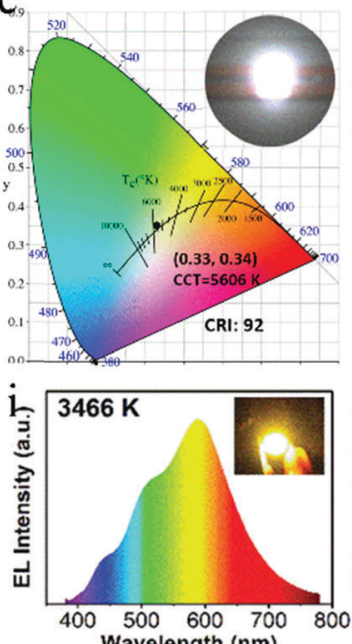

$400 \begin{gathered}500 \\ \text { Wavelength }(\mathrm{nm})\end{gathered}$

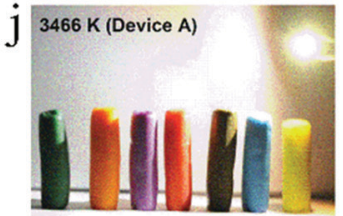

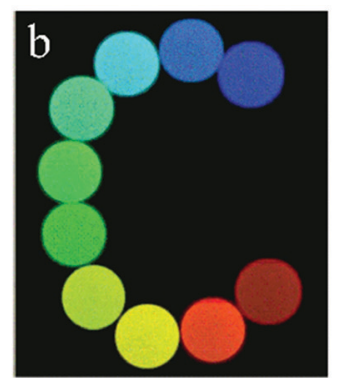
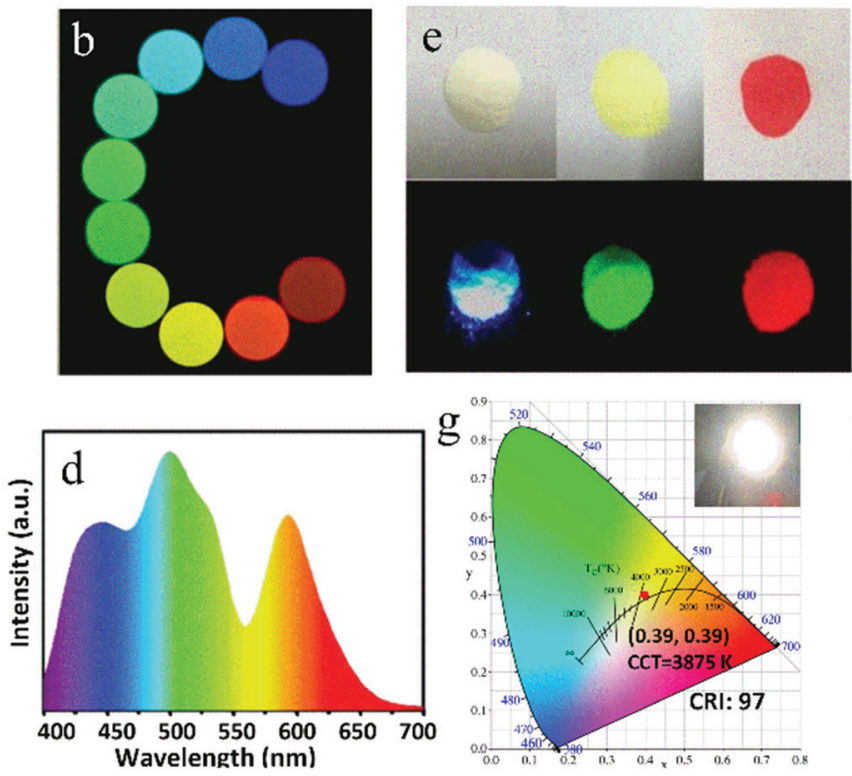

h
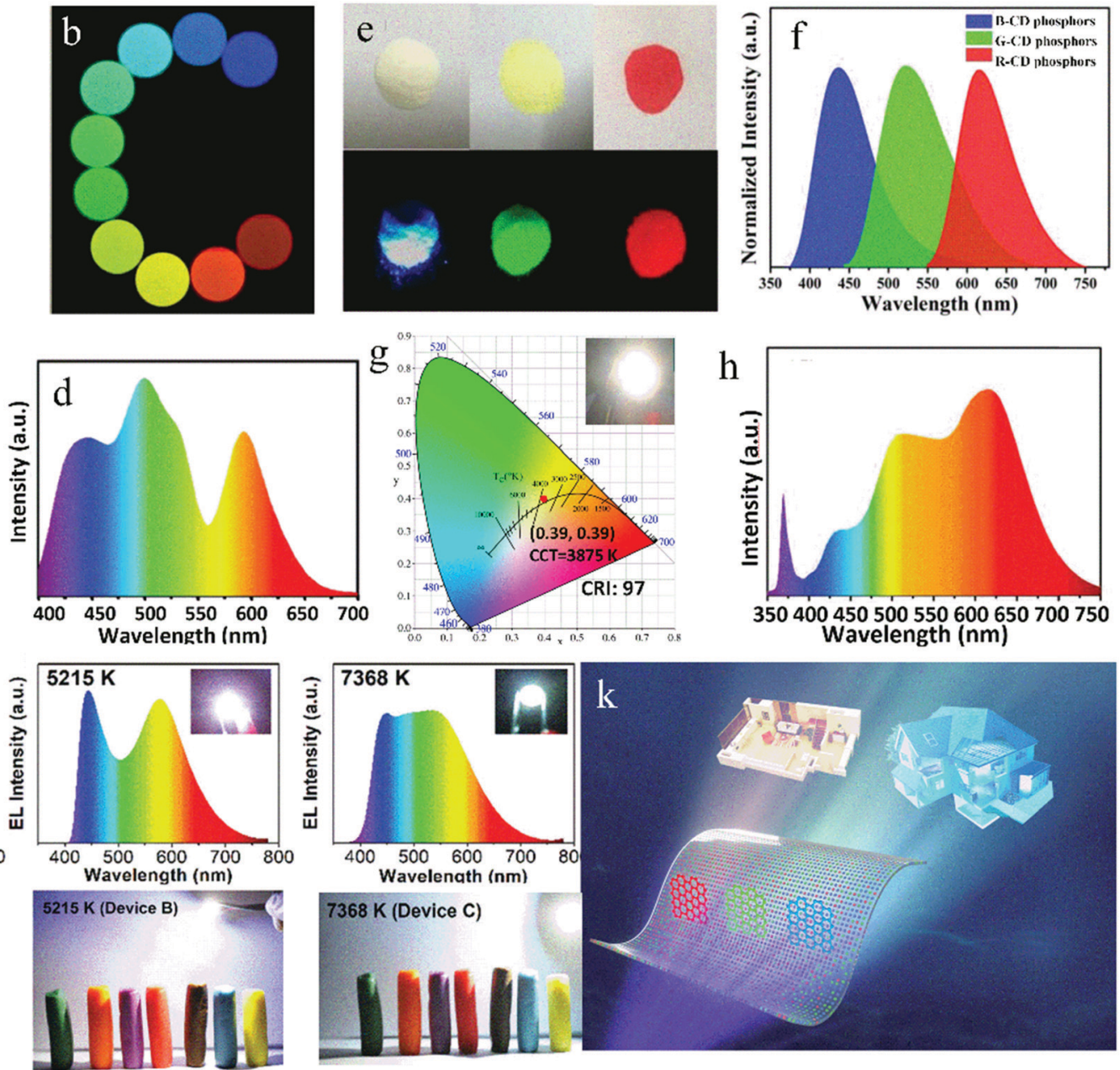

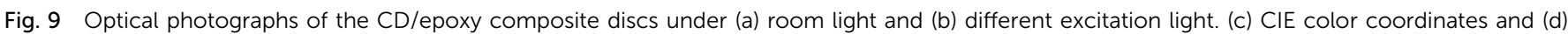

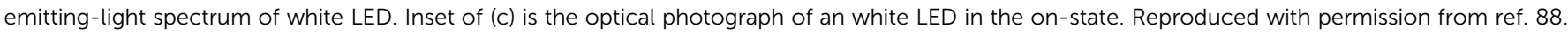

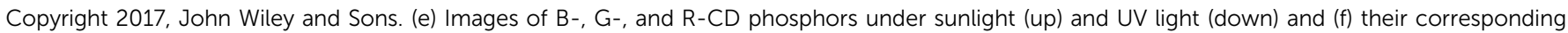

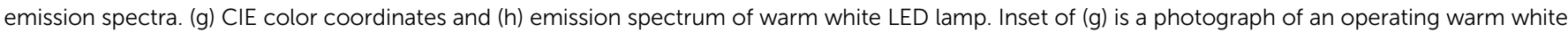

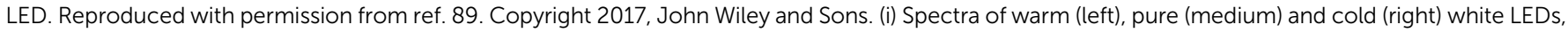

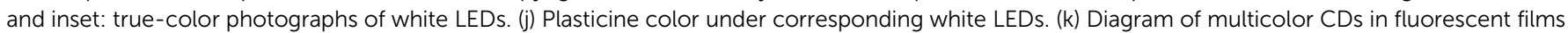
for white LED with an adjustable CCT. Reproduced with permission from ref. 90. Copyright 2019, The Royal Society of Chemistry.

characteristics. By adjusting the ratio of trichromatic CDs, LEDs with high CRI and adjustable CCT were obtained, as demonstrated in the PL spectra (Fig. 9i) and working diagram (Fig. 9j). The white LED with adjustable color temperature can satisfy the need of both indoor and outdoor lighting (Fig. 9k, back cover).

5.2.2 The application of solid-state fluorescent CDs as light-emitting layer in QLEDs. Because of their excellent electroluminescence performance, such as high color purity, good stability, and tunable color, QLEDs have become a new star in the field of lighting and display. CDs not only exhibit the excellent luminescence performances of traditional QDs, but also have the advantages of eco-friendliness, cost-effectiveness and simple process, making them stand out as novel QLED light-emitting layers. Presently, CD-based QLEDs are still in their infancy, and there is still little research in this area. QLEDs have very strict requirements for the CDs as the light-emitting layer. Firstly, the QY of
CDs directly affects the luminance efficiency of QLEDs. Therefore, CDs with a high solid-state QY are necessary. Secondly, CDs are easy to aggregate, and it is difficult to form a uniform and dense luminous layer film, leading to a decrease in the QLED brightness. Therefore, the quality of the CD luminescent film is also very important. Thirdly, CDs need to have good carrier transmission ability, which can be guaranteed by less surface defects and high conjugation in CDs. ${ }^{91}$ Finally, the energy level structure of CDs determines the injection capacity of carriers, which can be improved by reducing the injection barrier.

Wang et $a l .{ }^{79}$ first demonstrated the feasibility of CDs as a single light-emitting layer for QLED. Firstly, CDs were synthesized using CA and cetylamine as raw materials and octadecene as the solvent, and their QY was up to $60 \%$ in solution. Then, a white QLED with the traditional sandwich structure was prepared using the CDs as the light-emitting layer, polyethyldithiophene: $\operatorname{poly}(p$-styrene sulfonic acid) as the cavity transport layer and 
1,3,5-triplet(1-phenyl-1 $H$-benzimidazol-2-yl)benzene as the electron transport layer. The maximum brightness of the QLED was $35 \mathrm{~cd} \mathrm{~m}^{-2}$. Yuan et al. ${ }^{92}$ synthesized multicolor CDs with a significant quantum size effect by the hydrothermal treatment of diaminonaphthalene and CA. With above multicolor CDs as the light-emitting layer, blue, green, yellow, orange and red QLEDs were prepared with brightness of 136, 93, 60, 65 and $12 \mathrm{~cd} \mathrm{~m}^{-2}$, respectively. In addition, green light-emitting CDs were combined with polyvinyl carbazole (PVK) to prepare a white light-emitting QLED with a maximum brightness of $2050 \mathrm{~cd} \mathrm{~m}^{-2}$.

To overcome the solid-state quenching of CDs and improve their poor compatibility with the luminescence layer, Xu et al. ${ }^{93,94}$ employed a host-guest doping method and used PVK as the host and oleophylic CDs as the dopant to obtain high-brightness blue light, yellow light, and white light CD-based QLED with the maximum brightness of $569.8,339.5$ and $455.2 \mathrm{~cd} \mathrm{~m}^{-2}$, respectively. It was proven that the host-guest doping method can improve the dispersion of the luminescence layer, inhibit the luminescence quenching, and greatly improve the brightness of QLEDs. Subsequently, Yuan et al. ${ }^{17}$ prepared multicolor CDs with a unique triangular structure using phloroglucinol as the raw material via the solvothermal method, and prepared a CDbased QLED with CDs and PVK as the composite light-emitting layer (Fig. 10a). The brightness of the blue, green, yellow, and red QLEDs was as high as 1882, 4762, 2784, and $2344 \mathrm{~cd} \mathrm{~m}^{-2}$ (Fig. 10b-e), respectively. This great breakthrough indicated that CD-based QLEDs have a good development prospect in the solid-state lighting and full-color display fields.

5.2.3 The application of solid-state fluorescent CDs as a gain medium or phosphor in LDs. LDs, a new generation of solid-state light source, have the advantages of high brightness, long life, and stable performance. They are especially suitable for some special lighting fields (such as laser vehicle lights and projection display), and are expected to replace high power light sources, thus achieving energy conservation and environmental protection. Because LDs have high energy and a concentrated beam, when a laser light source irradiates on the surface of phosphors, a large amount of heat can be generated, which leads to the rapid weight loss and fluorescence quenching of phosphors. Therefore, the phosphor applied in LDs must have an excellent thermal- and photo-stability. Presently, most of the fluorescent materials used in LDs are rare earth-doped phosphors. ${ }^{95}$ However, rare earth-doped phosphors, originating from non-renewable resources, are limited by their high price, and thus it is urgent to develop new types of phosphors to replace the traditional rare earth phosphors. CDs have the advantages of excellent luminescence characteristics, diverse
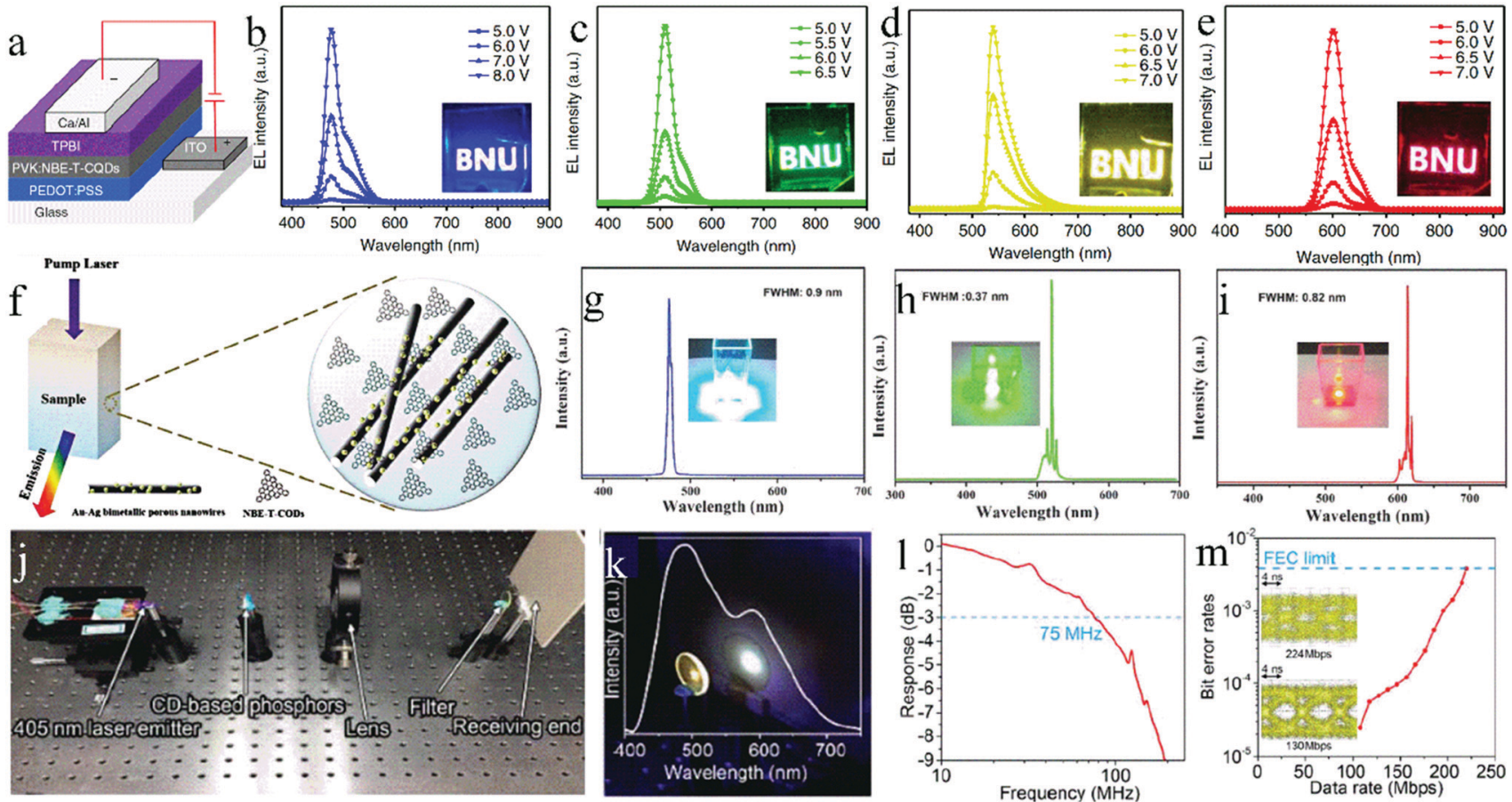

Fig. 10 (a) Device structure of the CD-based QLEDs. EL spectra of the B- (b), G- (c), Y- (d), and R-QLEDs (e) at different bias voltages. (Insets are the operation photographs of the B-, G-, Y-, and R-QLEDs with the logo of BNU). Reproduced with permission from ref. 17. Copyright 2018, Springer Nature. (f) Schematic illustration of the random lasing from the CD/Au-Ag bimetallic porous nanowire composites. (g) Blue, (h) green, and (i) red random lasing emission spectra from the $\mathrm{CD} / \mathrm{Au}-\mathrm{Ag}$ bimetallic porous nanowire composites excited by the 355, 480, and $532 \mathrm{~nm}$ laser pulses with a corresponding low pump energy density of $0.125,0.075$, and $0.057 \mathrm{~mJ} \mathrm{~cm}^{-2}$, respectively. The insets are photographs of the blue, green, and red random lasing emission, respectively. Reproduced with permission from ref. 98. Copyright 2018, John Wiley and Sons. (j) Experimental setup of the VLC link. (k) Photograph and the corresponding spectrum of the VLC generated white light using a $405 \mathrm{~nm}$ laser diode, CD-based phosphor, and a cut-off $420 \mathrm{~nm}$ filter. (l) Frequency response of the $-3 \mathrm{~dB}$ modulation bandwidth of the output white light cut off by a $420 \mathrm{~nm}$ filter. $(\mathrm{m})$ Bit error rate at different data rates using on-off keying of the white light. Reproduced with permission from ref. 102. Copyright 2019, The Royal Society of Chemistry. 
sources of raw materials, and environmental friendliness, showing great application potential in the field of LDs. ${ }^{96}$

Initially, CDs were used as a laser gain medium for LDs. Zhang et al. ${ }^{97}$ prepared CDs with a QY of $68 \%$ from CA and the silane coupling agent A2120, and dispersed the CDs into epoxy resin as a gain medium to realize laser emission. Qu et al. ${ }^{22}$ prepared green CDs from CA and urea and then realized green laser emission by using a CD ethanol solution as the gain medium. Yuan et al. ${ }^{98}$ optimized the reaction conditions to synthesize narrow band emission blue, green and red CDs using phloroglucinol as the raw material and ethanol as the solvent. These three classes of CDs were used as gain media to realize panchromatic lasers for the first time (Fig. 10f). Specifically, highly efficient blue, green, and red random lasers were obtained (Fig. 10g-i), respectively. In addition, Xi et al. ${ }^{99}$ mixed blue-, green- and red-emissive CDs with a concentration of $0.15,0.15,0.25 \mathrm{mg} \mathrm{mL}^{-1}$ at a ratio of $1: 3: 4$ as a gain medium, and then added $\mathrm{Au}-\mathrm{Ag}$ bimetallic nanowires as scattering particles to realize a white laser for the first time.

In recent years, researchers have used CDs as phosphors for LDs. Chen and Feng et al. ${ }^{100}$ combined blue-emissive CDs with green- and red-emissive lanthanide complexes, and embedded them in a PMMA matrix. By adjusting the proportion of these three components, a white light emission fluorescence film with high transparency was obtained. Based on this film, a white light LD was created with CIE of $(0.31,0.32)$ under $400 \mathrm{~nm}$ laser irradiation. Zhou et al. ${ }^{46}$ excited CDs with green emission as a single phosphor by a $450 \mathrm{~nm}$ blue light laser to obtain a white light laser, which further demonstrated the feasibility of CDs as a phosphor for LDs.

\subsection{Visible light communication}

Visible light communication (VLC) is new technology for transmitting data with high speed, which can realize "there is light, there is internet" using LED or LD lighting. Basically, VLC is a combination of lighting and communication. VLC has the advantages of high transmission efficiency rate, strong antiinterference ability, no electromagnetic radiation, high safety, low-carbon, and low cost. It can realize near-zero energy communication and effectively avoid electromagnetic signal leakage of radio communication. Therefore, VLC has gradually become a research hotspot in the field of wireless communication. The commercial rare earth phosphors have a long fluorescence lifetime, leading to a narrow bandwidth of VLC, which limits the transmission rate of VLC. In fact, a shorter fluorescence lifetime will result in a more sensitive response, higher limit transmission rate, and closer modulation bandwidth with the light source, which is meaningful for VLC. Therefore, phosphors with a short fluorescence lifetime are popular in the field of VLC. Compared with other fluorescent materials, CDs have a shorter fluorescence lifetime, and therefore are highly applicable in the field of VLC. ${ }^{19}$

Zhou et al. $^{46}$ obtained a green light-emitting CD phosphor with a solid-state QY of $25 \%$ through the oxidation of CDs with $\mathrm{H}_{2} \mathrm{O}_{2}$. Then, a $442 \mathrm{~nm}$ blue diode laser was used as the excitation source to obtain white light-emissive VCL with a bandwidth of $285 \mathrm{MHz}$ and a transmission speed of 435 Mbps. Tian et al. ${ }^{101}$ used CA and urea as raw materials, and water, glycerin and DMF as the solvent to synthesize blue-, green- and red-emissive CDs, respectively. The fluorescence lifetime of these three classes of CDs was 6.8-8.8 ns, much shorter that of rare earth phosphors, organic fluorescent materials, and perovskite quantum dots. Based on the above work, they ${ }^{102}$ obtained a white-emissive CD phosphor by the combination of the blue-, green- and red-emissive CDs, and then dispersed them in sodium silicate to prevent their aggregation (Fig. 10j and k). Finally, VCL was realized with a bandwidth of $75 \mathrm{MHz}$ and transmission rate of $224 \mathrm{Mbps}$ (Fig. 10l and $\mathrm{m}$ ), superior to that of the traditional nitride phosphor $(12.4 \mathrm{MHz})$ and commercial YAG phosphors (3-12 MHz). ${ }^{103}$

\section{Conclusion and prospect}

In summary, we introduced in depth and in detail the research status of solid-state fluorescent CDs, including the quenchingresistant strategy, high quantum efficiency control method, multicolor regulation method, and application, and arrived at the following conclusions. (1) On the one hand, CD nanoparticles can be effectively separated to increase their space distance, thereby inhibiting the fluorescence quenching by dispersing them in a matrix, synthesizing self-dispersive CDs in situ with a precursor with a long chain structure, or synthesizing CDs with strong electrostatic repulsion, hydrogen bonds, or low conjugation. On the other hand, synthesizing CDs with uniform surface states or uniform particle sizes can reduce their aggregation and also inhibit their fluorescence quenching. (2) CDs with high solid-state quantum efficiency can be realized by controlling the matrix type and CD concentration, doping heteroatoms, and adding salt crystals in situ and reducing the carbonization degree. (3) Multicolor solid-state fluorescent CDs can be obtained not only by the effective dispersion of multicolor CDs in a matrix, but also by the gradient concentration dispersion of CDs with concentrationinduced luminescence redshift by post-processing or in situ embedding within a matrix. In addition, the luminescence center of self-quenching CDs can be changed by controlling the degree of cross-linking carbonization of raw materials, and the luminescence color of CDs can also be accordingly regulated. (4) Solid-state fluorescent CDs show broad application prospects in the fields of fingerprint identification, LED, QLED, LD and other photoelectric devices, and VCL.

However, there are still some challenges with the fluorescence characteristics of solid-state CDs to be further explored in future research. (1) Revealing the internal relationship between the structure and luminescence of CDs. Presently, for most solid-state CDs, their QY decreases significantly with an increase in the luminescence wavelength. Therefore, it is important to realize the coordinated regulation of the solidstate QY and emission wavelength. Elucidating the correlation between the structure and aggregation state of CDs and their solid-state QY and luminescence wavelength, and establishing 
the relevant internal mechanism will guide the synthesis of high QY multicolor solid-state fluorescent CDs. (2). Exploring methods for the preparation of CDs with consistent luminescence in the dispersed state and aggregated state. Presently, most CDs show obvious concentration-dependent luminescence characteristics. However, the spectral shift in the solid state usually occurs with competitive nonradiative decay, which leads to a decrease in QY. In addition, the change in emission color needs an additional optimization process to obtain appropriate emission colors in the solid state for the fabrication of optoelectronics. Therefore, it is also very important to develop CDs with consistent luminescence in the solution and solid states. The following methods are effective for consistent luminescent in CDs: decreasing the degree of cross-linking carbonization between the reactants, and modifying ligands on the surface of CDs to create steric hindrance between their luminescence centers. (3) Expanding the application of solid-state fluorescent CDs in the electroluminescence field. In recent years, CD-based QLEDs have attracted increasing attention from researchers, but the brightness and efficiency of QLEDs are far from the level of commercialization. The luminescent layer material plays a crucial role in the performance of QLEDs. Thus, it is necessary to rationally design the structure of CDs so that they not only have high solid-state QY, but also good film-forming property and excellent carrier transmission and injection capability.

\section{Conflicts of interest}

There are no conflicts to declare.

\section{Acknowledgements}

This work was financially supported by the National Natural Science Foundation of China (U1710117 and 51972221) and Research Project Supported by Shanxi Scholarship Council of China (2020-051 and HGKY2019027).

\section{References}

1 Y. P. Sun, B. Zhou, Y. Lin, W. Wang, K. A. S. Fernando, P. Pathak, M. J. Meziani, B. A. Harruff, X. Wang and H. Wang, J. Am. Chem. Soc., 2006, 128, 7756-7757.

2 S. Zhu, Y. Song, X. Zhao, J. Shao, J. Zhang and Y. Bai, Nano Res., 2015, 8, 355-381.

3 Z. Kang and S. T. Lee, Nanoscale, 2019, 11, 19214-19224.

4 B. Li, Y. Guo, A. Iqbal, Y. Dong, W. Li, W. Liu, W. Qin and Y. Wang, RSC Adv., 2016, 6, 107263.

5 A. L. Himaja, P. S. Karthik and S. P. Singh, Chem. Rec., 2015, 15, 595.

6 X. Xu, R. Ray, Y. Gu, H. J. Ploehn, L. Gearheart, K. Raker and W. A. Scrivens, J. Am. Chem. Soc., 2004, 126, 12736-12737.

7 X. Wang, L. Cao, F. Lu, M. J. Meziani, H. Li, G. Qi, B. Zhou, B. A. Harruff, F. Kermarrec and Y. P. Sun, Chem. Commun., 2009, 3774-3776.
8 S. T. Yang, L. Cao, P. G. Luo, F. Lu, X. Wang, H. Wang, M. J. Meziani, Y. Liu, G. Qi and Y. P. Sun, J. Am. Chem. Soc., 2009, 131, 11308-11309.

9 Q. L. Zhao, Z. L. Zhang, B. H. Huang, J. Peng, M. Zhang and D. W. Pang, Chem. Commun., 2008, 5116-5118.

10 L. Zheng, Y. Chi, Y. Dong, J. Lin and B. Wang, J. Am. Chem. Soc., 2009, 131, 4564.

11 A. B. Bourlinos, A. Stassinopoulos, D. Anglos, R. Zboril, M. Karakassides and E. P. Giannelis, Small, 2008, 4, 455-458.

12 Y. Liu, N. Xiao, N. Gong, H. Wang, X. Shi, W. Gu and L. Ye, Carbon, 2014, 68, 258-264.

13 P. Zhao, X. Li, G. Baryshnikov, B. Wu, H. Ågren, J. Zhang and L. Zhu, Chem. Sci., 2018, 9, 1323-1329.

14 A. E. Tomskaya, M. N. Egorova, A. N. Kapitonov, D. V. Nikolaev, V. I. Popov, A. L. Fedorov and S. A. Smagulova, Phys. Status Solidi B, 2018, 255, 1700222.

15 Q. T. Huang, X. F. Lin, X. M. Li, W. Wen, L. P. Lin and S. R. Hu, Prog. Chem., 2015, 27, 1604-1614.

16 H. J. Wang, T. T. Yu, H. L. Chen, W. B. Nan, L. Q. Xie and Q. Q. Zhang, Dyes Pigm., 2018, 159, 245-251.

17 F. Yuan, Y. Ting, S. L. Z. Sui, Z. B. Wang, Z. F. Xi, Y. C. Li, X. H. Li, L. Z. Fan, Z. A. Tian, A. M. Chen, M. X. Jin and S. H. Yang, Nat. Commun., 2018, 9, 1-11.

18 J. Ren, L. Stagi and P. Innocenzi, Prog. Solid State Chem., 2020, 100295, DOI: 10.1016/ j.progsolidstchem.2020.100295.

19 B. S. Xu, J. C. Xu, J. X. Zheng, Y. Z. Yang and X. G. Liu, Chin. Sci. Bull., 2019, 64, 1441-1455.

20 J. Zhu, X. Bai, X. Chen, Z. Xie, Y. Zhu, G. Pan, Y. Zhai, H. Zhang, B. Dong and H. Song, Dalton Trans., 2018, 47, 3811-3818.

21 P. Long, Y. Feng, Y. Li, C. Cao, S. Li, H. An, C. Qin, J. Han and W. Feng, ACS Appl. Mater. Interfaces, 2017, 9, 37981-37990.

22 M. Sun, S. Qu, Z. Hao, W. Ji, P. Jing, H. Zhang, L. Zhang, J. Zhao and D. Shen, Nanoscale, 2014, 6, 13076-13081.

23 T. H. Kim, F. Wang, P. McCormick, L. Wang, C. Brown and Q. Li, J. Lumin., 2014, 154, 1-7.

24 D. Zhou, Y. Zhai, S. Qu, D. Li, P. Jing, W. Ji, D. Shen and A. L. Rogach, Small, 2016, 13, 1602055.

25 J. Wang, F. Zhang, Y. Wang, Y. Yang and X. Liu, Carbon, 2018, 126, 426-436.

26 W. K. Kipnusu, C. Doñate-Buendía, M. Fernández-Alonso, J. Lancis and G. Mínguez-Vega, Part. Part. Syst. Charact., 2020, 37, 2000093.

27 Z. Ding, L. Di, P. Jing, Y. Zhai, D. Shen, S. Qu, A. L. Rogach, Z. Ding, L. Di and P. Jing, Chem. Mater., 2017, 29, 1779-1787.

28 T. Feng, S. Zhu, Q. Zeng, S. Lu, S. Tao, J. Liu and B. Yang, ACS Appl. Mater. Interfaces, 2017, 10, 12262-12277.

29 D. Xu, F. Lei, H. Chen, L. Yin, Y. Shi and J. Xie, RSC Adv., 2019, 9, 8290-8299.

30 E. Liu, D. Li, X. Zhou, G. Zhou, H. Xiao, D. Zhou, P. Tian, R. Guo and S. Qu, ACS Sustainable Chem. Eng., 2019, 7, 9301-9308. 
31 Y. Chen, M. Zheng, Y. Xiao, H. Dong, H. Zhang, J. Zhuang, H. Hu, B. Lei and Y. Liu, Adv. Mater., 2016, 28, 312-318.

32 B. P. Jiang, Y. X. Yu, X. L. Guo, Z. Y. Ding, B. Zhou, H. Liang and X. C. Shen, Carbon, 2018, 128, 12-20.

33 F. Wang, Z. Xie, H. Zhang, C. Y. Liu and Y. G. Zhang, Adv. Funct. Mater., 2011, 21, 1027-1031.

34 J. Zheng, Y. Wang, Z. Feng, Y. Yang, X. Liu, K. Guo, W. Hua and B. Xu, J. Mater. Chem. C, 2017, 5, 9629-9637.

35 J. He, Y. He, Y. Chen, B. Lei, J. Zhuang, Y. Xiao, Y. Liang, M. Zheng, H. Zhang and Y. Liu, Small, 2017, 13, 1700075.

36 C. L. Shen, J. H. Zang, Q. Lou, L. X. Su, Z. Li, Z. Y. Liu, L. Dong and C. X. Shan, Carbon, 2018, 136, 359-368.

37 D. Wang, W. U. Khan and Y. Wang, Chem. - Asian J., 2019, 14, 286-292.

38 M. Xu, G. He, Z. Li, F. He, F. Gao, Y. Su, L. Zhang, Z. Yang and Y. Zhang, Nanoscale, 2016, 6, 10307-10315.

39 Y. N. Zheng, J. X. Zheng, J. L. Wang, Y. Z. Yang, T. P. Lu and X. G. Liu, Nanomaterials, 2020, 10, 303.

40 T. Meng, T. Yuan, X. Li, Y. Li, L. Fan and S. Yang, Chem. Commun., 2019, 55, 6531-6534.

41 C. Shen, J. Wang, Y. Cao and Y. Lu, J. Mater. Chem. C, 2015, 3, 6668-6675.

42 Y. Choi, B. Kang, J. Lee, S. Kim, G. T. Kim, H. Kang, B. R. Lee, H. Kim, S.-H. Shim and G. Lee, Chem. Mater., 2016, 28, 6840-6847.

43 T. Feng, Q. Zeng, S. Lu, X. Yan, J. Liu, S. Tao, M. Yang and B. Yang, ACS Photonics, 2017, 5, 502-510.

44 X. M. Li, Y. L. Liu, X. F. Song, H. Wang, H. S. Gu and H. B. Zeng, Angew. Chem. Int. Ed., 2015, 127, 1779-1784.

45 D. Li, D. Han, S. N. Qu, L. Liu, P. T. Jing, D. Zhou and W. Y. Ji, Light: Sci. Appl., 2016, 5, 93-100.

46 J. Z. Zhou, P. F. Tian, X. Y. Liu, S. L. Mei, D. Zhou, D. Li, P. T. Jing, W. L. Zhang, R. Q. Guo, S. N. Qu and A. L. Rogach, Adv. Sci., 2018, 5, 1800369.

47 Y. Zhang, P. Zhuo, H. Yin, Y. Fan, J. Zhang, X. Liu and Z. Chen, ACS Appl. Mater. Interfaces, 2019, 11, 24395-24403.

48 J. Zhou, H. Zhou, J. Tang, S. Deng, F. Yan, W. Li and M. Qu, Microchim. Acta, 2016, 184, 343-368.

49 L. Li and T. Dong, J. Mater. Chem. C, 2018, 6, 7944-7970.

50 Y. Deng, X. Chen, F. Wang, X. Zhang, D. Zhao and D. Shen, Nanoscale, 2014, 6, 10388-10393.

51 K. Jin, J. Zhang, W. Tian, X. Ji, J. Yu and J. Zhang, ACS Sustainable Chem. Eng., 2020, 8, 5937-5945.

52 Z. Xie, F. Wang and C. Y. Liu, Adv. Mater., 2012, 24, 1716-1721. 53 D. Zhou, D. Li, P. Jing, Y. Zhai, D. Shen, S. Qu and A. L. Rogach, Chem. Mater., 2017, 29, 1779-1787.

54 S. Tao, S. Lu, Y. Geng, S. Zhu, S. A. T. Redfern, Y. Song, T. Feng, W. Xu and B. Yang, Angew. Chem., Int. Ed., 2018, 57, 2393-2398.

55 J. Y. Wei, Q. Lou, J. H. Zang, Z. Y. Liu, Y. L. Ye, C. L. Shen, W. B. Zhao, L. Dong and C. X. Shan, Adv. Opt. Mater., 2020, 8, 1901938.

56 H. J. Yoo, B. E. Kwak and D. H. Kim, J. Phys. Chem. C, 2019, 123, 27124-27131.

57 Y. Zhang, C. Li, Y. Fan, C. Wang, R. Yang, X. Liu and L. Zhou, Nanoscale, 2016, 8, 19744-19753.
58 C. Wang, T. Hu, Y. Y. Chen, Y. L. Xu and Q. J. Song, ACS Appl. Mater. Interfaces, 2019, 11, 22332-22338.

59 S. K. Bhunia, S. Nandi, J. Manna, R. Shikler and R. Jelinek, Nanoscale, 2016, 8, 3400-3406.

60 T. Song, Y. Zhao, K. Matras-Postolek and P. Yang, J. Lumin., 2019, 212, 69-75.

61 S. Lai, Y. Jin, L. Shi, R. Zhou, Y. Zhou and D. An, Nanoscale, 2020, 12, 591-601.

62 X. Meng, Q. Chang, C. Xue, J. Yang and S. Hu, Chem. Commun., 2017, 53, 3074-3077.

63 X. X. Ba, L. Zhang, Y. L. Yin, F. L. Jiang, P. Jiang and Y. Liu, J. Colloid Interface Sci., 2020, 565, 77-85.

64 Y. Chen, H. Lian, Y. Wei, X. He, Y. Chen, B. Wang, Q. Zeng and J. Lin, Nanoscale, 2018, 10, 6734-6743.

65 F. Yan, Y. Jiang, X. Sun, J. Wei, L. Chen and Y. Zhang, Nano Res., 2019, 13, 52-60.

66 B. E. Kwak, H. J. Yoo and D. H. Kim, Adv. Opt. Mater., 2019, 7, 1900932.

67 J. Zhu, H. Chu, T. Wang, C. Wang and Y. Wei, Microchem. J., 2020, 158, 105142.

68 J. Shao, S. Zhu, H. Liu, Y. Song, S. Tao and B. Yang, Adv. Sci., 2017, 4, 1700395.

69 Y. Zhan, B. Shang, M. Chen and L. Wu, Small, 2019, 15, 1901161.

70 Q. Chang, Y. Ding, S. Cheng, W. Shen, Z. Zhou, Y. Yin, T. Sun, C. Ban, Z. Deng, J. Liu, F. Xiu and W. Huang, Nanoscale, 2019, 11, 2131-2137.

71 S. Cheng, Y. Ding, Q. Chang, S. Zhong, W. Shen, H. Mao, X. Zhai, G. Lu, Z. Deng, J. Liu, F. Xiu and W. Huang, Nanoscale, 2019, 11, 14592-14597.

72 C. L. Shen, J. H. Zang, Q. Lou, L. X. Su, Z. Li, Z. Y. Liu, L. Dong and C. X. Shan, Carbon, 2018, 136, 359-368.

73 H. Song, X. Liu, B. Wang, Z. Tang and S. Lu, Sci. Bull., 2019, 64, 1788-1794.

74 J. Yang, Y. Liu, J. Wang, S. Wang, X. Zhou and H. Li, J. Mater. Chem. C, 2019, 7, 7806-7811.

75 J. Zhu, X. Bai, X. Chen, H. Shao, Y. Zhai, G. Pan, H. Zhang, E. V. Ushakova, Y. Zhang, H. Song and A. L. Rogach, Adv. Opt. Mater., 2019, 7, 1801599.

76 D. Zhou, P. Jing, Y. Wang, Y. Zhai, D. Li, Y. Xiong, A. V. Baranov, S. Qu and A. L. Rogach, Nanoscale Horiz., 2019, 4, 388-395.

77 J. Chen, J. S. Wei, P. Zhang, X. Q. Niu, W. Zhao, Z. Y. Zhu, H. Ding and H. M. Xiong, ACS Appl. Mater. Interfaces, 2017, 9, 18429-18433.

78 M. Tuerhong, X. U. Yang, Y. X. Bo, K. University and N. University, Chin. J. Anal. Chem., 2017, 45, 139-150.

79 F. Wang, Y. H. Chen, C. Y. Liu and D. G. Ma, Chem. Commun., 2011, 47, 3502-3504.

80 D. Fernandes, M. J. Krysmann and A. Kelarakis, Chem. Commun., 2015, 51, 4902-4905.

81 C. Wang, J. Zhou, L. L. Lu and Q. Song, Part. Part. Syst. Charact., 2018, 35, 1700387.

82 R. Bandi, H. G. Kannikanti, R. dadigala, B. R. Gangapuram, J. R. Vaidya and V. Guttena, Opt. Mater., 2020, 109, 110349.

83 E. F. Schubert and J. K. Kim, Science, 2005, 308, 1274-1278. 
84 X. Guo, C. F. Wang, Z. Y. Yu, L. Chen and S. Chen, Chem. Commun., 2012, 48, 2692.

85 F. Zhang, X. T. Feng, Y. Zhang, L. P. Yan, Y. Z. Yang and X. G. Liu, Nanoscale, 2016, 8, 8618.

86 Y. T. Xie, J. X. Zheng, Y. L. Wang, J. L. Wang, Y. Z. Yang and X. G. Liu, Nanotechnology, 2019, 30, 085406.

87 Y. Wang, K. Wang, Z. Han, Z. Yin, C. Zhou, F. Du, S. Zhou, P. Chen and Z. Xie, J. Mater. Chem. C, 2017, 5, 9629-9637.

88 X. Miao, D. Qu, D. Yang, B. Nie, Y. Zhao, H. Fan and Z. Sun, Adv. Mater., 2017, 30, 1704740.

89 Z. Wang, F. Yuan, X. Li, Y. Li, H. Zhong, L. Fan and S. Yang, Adv. Mater., 2017, 29, 1702910.

90 Y. Ding, J. Zheng, J. Wang, Y. Yang and X. Liu, J. Mater. Chem. C, 2019, 7, 1502-1509.

91 Y. Ding, F. Zhang, J. Xu, Y. Miao, Y. Yang, X. Liu and B. Xu, RSC Adv., 2017, 7, 28754-28762.

92 F. Yuan, Z. B. Wang, X. H. Li, Y. C. Li, Z. A. Tian, L. Fan and S. Yang, Adv. Mater., 2017, 29, 1604436.

93 J. Xu, Y. Miao, J. Zheng, Y. Z. Yang and X. G. Liu, Adv. Opt. Mater., 2018, 6, 1800181.
94 J. Xu, Y. Miao, J. Zheng, H. Wang, Y. Z. Yang and X. G. Liu, Nanoscale, 2018, 10, 11211.

95 H. Y. Ryu and D. H. Kim, J. Opt. Soc. Korea, 2010, 14, 415-419.

96 W. F. Zhang, H. Zhu, S. F. Yu and H. Y. Yang, Adv. Mater., 2012, 24, 2263-2267.

97 W. F. Zhang, L. M. Jin, S. F. Yu, H. Zhu, S. S. Pan, Y. H. Zhao and H. Y. Yang, J. Mater. Chem. C, 2014, 2, 1525-1531.

98 F. Yuan, Z. Xi, X. Shi, Y. Li, X. Li, Z. Wang, L. Fan and S. Yang, Adv. Opt. Mater., 2019, 7, 1801202.

99 Z. Xi, F. Yuan, Z. Wang, S. Li and L. Fan, Acta Chim. Sin., 2018, 76, 460.

100 B. Chen and J. Feng, J. Phys. Chem. C, 2015, 119, 7865-7872.

101 Z. Tian, X. Zhang, D. Li, D. Zhou, P. Jing, D. Shen, S. Qu, R. Zboril and A. L. Rogach, Adv. Opt. Mater., 2017, 5, 1700416.

102 Z. Tian, P. Tian, X. Zhou, G. Zhou, S. Mei, W. Zhang, X. Zhang, D. Li, D. Zhou, R. Guo, S. Qu and A. L. Rogach, Nanoscale, 2019, 11, 3489-3494.

103 D. Tsonev, H. Chun, S. Rajbhandari, J. J. D. Mckendry and D. O’Brien, IEEE Photon. Technol. Lett., 2014, 26, 637-640. 